\title{
Generalizable approaches for genomic prediction of metabolites in plants
}

2 Lauren J. Brzozowski ${ }^{1 *}$, Malachy T. Campbell ${ }^{1}$, Haixiao Hu${ }^{1}$, Melanie Caffe ${ }^{2}$, Lucía Gutiérrez ${ }^{3}$,

3 Kevin P. Smith ${ }^{4}$, Mark E. Sorrells ${ }^{1}$, Michael A. Gore ${ }^{1}$, and Jean-Luc Jannink ${ }^{1,5}$

$4 \quad{ }^{1}$ Plant Breeding and Genetics Section, School of Integrative Plant Science, Cornell University,

5 Ithaca, NY 14853, USA

$6{ }^{2}$ Department of Agronomy, Horticulture \& Plant Science, South Dakota State University,

7 Brookings, SD 57006, USA

$8{ }^{3}$ Department of Agronomy University of Wisconsin-Madison Madison, WI 53706, USA

$9{ }^{4}$ Department of Agronomy \& Plant Genetics University of Minnesota St. Paul, MN 55108, USA

$10{ }^{5}$ USDA-ARS, Robert W. Holley Center for Agriculture and Health, Ithaca, NY 14853 USA

13 Abbreviations: drBLUPs, deregressed best linear unbiased predictors; GC-MS, gas

14 chromatography - mass spectrometry; LC-MS, liquid chromatography - mass spectrometry;

15 MEP, Methylerythritol Phosphate pathway; mGWAS, metabolite genome wide association

16 study; MVA, Mevalonate Acid pathway 


\section{ABSTRACT}

18 Plant metabolites are important for plant breeders to improve nutrition and agronomic

19 performance, yet integrating selection for metabolomic traits is limited by phenotyping expense

20 and limited genetic characterization, especially of uncommon metabolites. As such, developing

21 biologically-based and generalizable genomic selection methods for metabolites that are

22 transferable across plant populations would benefit plant breeding programs. We tested genomic

23 prediction accuracy for more than 600 metabolites measured by GC-MS and LC-MS in oat

24 (Avena sativa L.) seed. Using a discovery germplasm panel, we conducted metabolite GWAS

25 (mGWAS) and selected loci to use in multi-kernel models that encompassed metabolome-wide

26 mGWAS results, or mGWAS from specific metabolite structures or biosynthetic pathways.

27 Metabolite kernels developed from LC-MS metabolites in the discovery panel improved

28 prediction accuracy of LC-MS metabolite traits in the validation panel, consisting of more

29 advanced breeding lines. No approach, however, improved prediction accuracy for GC-MS

30 metabolites. We tested if similar metabolites had consistent model ranks and found that, while

31 different metrics of 'similarity' had different results, using annotation-free methods to group

32 metabolites led to consistent within-group model rankings. Overall, testing biological rationales

33 for developing kernels for genomic prediction across populations, contributes to developing

34 frameworks for plant breeding for metabolite traits. 


\section{INTRODUCTION}

37 Plant metabolites contribute to human health, food flavor, and plant resistance to stresses, and

38 thus are important traits for plant breeders (Kumar et al., 2017; Zhu et al., 2019). While selection

39 for some metabolites is possible through correlated traits, like color, many metabolites are

40 phenotyped through metabolomics approaches like chromatography and mass spectrometry

41 (Fernie \& Tohge, 2017). Some key challenges in plant breeding for metabolites are the diversity

42 of plant metabolites, with hundreds of thousands predicted (Afendi et al., 2012), a generally

43 limited knowledge of the genetic architecture of metabolite traits (Soltis \& Kliebenstein, 2015),

44 and expense in generating metabolomics data. As our capacity to measure and identify plant

45 metabolites grows (Fernie \& Tohge, 2017), developing biologically-based and generalizable

46 selection methods that are transferable across plant populations would benefit plant breeding

47 programs.

Most knowledge of the genetic bases of metabolite variation in crops comes from models

49 like tomato, maize, and rice, and nutritional metabolites, such as vitamin precursors (Luo, 2015;

$50 \quad$ Fernie \& Tohge, 2017; Wager \& Li, 2018). While this work encompasses biochemical pathways

51 that are largely conserved, there is also a growing body of work on specialized metabolites,

52 metabolites that contribute to ecological interactions and are generally restricted to few lineages,

53 for instance, alkaloid production in tomato (Zhu et al., 2018) and benzoxazinoid production in

54 maize (Zhou et al., 2019). Together, these studies have shaped our understanding of the genetic

55 architecture of plant metabolite traits: while some specialized metabolites have oligogenic

56 genetic architecture (Diepenbrock et al., 2017, 2021), many loci contributing to metabolite

57 variation have small effects, and there are multiple examples of balancing selection for

58 metabolites (Soltis \& Kliebenstein, 2015). Given the typically complex genetic architecture and 
59 small-effect loci that underpin metabolite traits, techniques like genomic prediction and selection

60 would be particularly useful methods to implement in plant breeding programs (Heffner et al.,

61 2009; Heslot et al., 2015).

62 Genomic prediction and selection studies have shown that metabolomic traits are viable

63 candidates for genomic selection. For instance, genomic selection on color (a proxy for

64 provitamin A) in winter squash (Cucurbita moschata) fruit, led to significant population

65 improvement over four cycles of selection (Hernandez et al., 2020). In addition, average

66 genomic prediction accuracy for measured vitamin metabolites was 0.43 for provitamin A in

67 maize kernels (Owens et al., 2014), and 0.49 for vitamin $\mathrm{E}$ in fresh sweet corn kernels (Baseggio

68 et al., 2019). Recently, others have also tested strategies for incorporating multiomic information

69 in prediction of metabolites. For instance, computing relationship matrices from metabolomics

70 data (Campbell et al., 2021a) or metabolomics and transcriptomics data (Hu et al., 2021) led to

71 high average prediction accuracies $(r>0.4)$ for fatty acid traits in oat (Avena sativa) seed. These

72 studies have demonstrated that genomic prediction is effective for a few to tens of biochemically

73 similar metabolites traits. Expanding to consider more metabolites would allow for an

74 understanding of the generalizability of the results. Further, as with much work involving

75 multiomic datasets, connecting genomic prediction results to biological mechanisms is a

76 challenge.

77 One approach to elucidate and incorporate biological bases into genomic prediction has

78 been through tests of genomic partitioning where, if the partitioned SNPs are enriched for causal

79 variants, prediction accuracy could be improved (Sarup et al., 2016). Recent work in genomic

80 prediction of 65 free amino acid metabolite traits in Arabidopsis seeds partitioned genomic SNPs

81 using annotations from 20 biochemical pathways, and found that inclusion of pathway SNPs as a 
82 kernel in a multikernel BLUP model improved prediction ability (Turner-Hissong et al., 2020).

83 In other examples, genomic prediction with pathway SNPs alone was equivalent to genome-wide

84 prediction for provitamin A compounds (carotenoids) in maize kernels (Owens et al., 2014), but

85 biosynthetic pathway SNPs performed worse than genome-wide SNPs for prediction of vitamin

86 E (tocochromanols) in fresh sweet corn kernels (Baseggio et al., 2019). These differences could

87 be due to the degree to which markers were in LD with causal variants (Baseggio et al., 2019) or

88 may point to causal variation being attributable to regulation (local or distal), or factors like

89 metabolite transport (Soltis \& Kliebenstein, 2015). Finally, while integrating prior information

90 about biochemical pathways has promising but mixed success, its application remains limited to

91 organisms with well annotated genomic, transcriptomic and metabolomic resources.

92 Strategies to conduct genomic partitioning without incorporating prior biosynthesis

93 information have also been tested. In oat (Avena sativa L.), a hexaploid with a recently available

94 whole genome sequence, (Campbell et al., 2021b) leveraged untargeted metabolomics data with

95 over 1600 metabolites to conduct factor analysis to uncover genomic regions that influence

96 metabolite composition. Using a multi-kernel approach, incorporating a kernel using GWAS

97 results of factors improved prediction accuracy of lipid and protein traits across populations

98 (Campbell et al., 2021b). In this analysis, factors were most commonly enriched for lipids which

99 perhaps contributed to increased prediction accuracy of fatty acids (a type of lipid), but it would

100 be intriguing to understand if this result is generalizable across more types of metabolites that

101 were less represented in the factor data set.

102 We sought to expand upon the work of (Campbell et al., 2021b) to test prediction models

103 for the entire oat seed metabolome and develop generalized genomic prediction method

104 frameworks. Oat seeds contain multiple healthful metabolites such as unsaturated fatty acids, 
105 beta-glucans, fiber as well as antioxidants (Stewart \& McDougall, 2014), and fatty acid traits

106 have been a target of GWAS (Carlson et al., 2019) and genomic prediction (Campbell et al.,

107 2021b; a; Hu et al., 2021). Using this well-studied germplasm, we examined more than 600

108 metabolites in oat seed measured by GC-MS and LC-MS and tested genomic prediction accuracy

109 using two-kernel models. Our objectives were to characterize the measured metabolome by

110 metabolite GWAS (mGWAS), leverage mGWAS results to select loci for two-kernel genomic

111 prediction models to test hypotheses about the most informative, biologically-based genome

112 partitioning methods of metabolomics data, and to evaluate prediction accuracy of these models

113 in a separate germplasm panel. To this end, we conducted mGWAS in a discovery panel and

114 generated kernels from significant mGWAS SNPs for any metabolite, or of metabolites

115 identified by structure as lipids or belonging to specific biosynthetic pathways thereof (terpenoid

116 biosynthesis pathways). Genomic prediction accuracy was evaluated in a validation germplasm

117 panel using K-fold cross validation. We hypothesized that kernels encompassing metabolome-

118 wide information would increase prediction accuracy for many metabolites, while kernels for

119 specific metabolite types or pathways would result in the highest prediction accuracy of their

120 own metabolites. We also hypothesized that similar metabolites would have similar genomic

121 prediction results (in terms of model rank), and defined metabolomic 'similarity' in three ways:

122 high-confidence annotations, structural annotations, or by an annotation-free method. Broadly, as

123 plant breeders target larger numbers and more diverse (less well known) metabolites, developing

124 frameworks for structuring genomic prediction models is important. This work tests different

125 biological rationales for incorporating information into genomic prediction, and transferability

126 across populations. 


\section{MATERIALS and METHODS}

\section{Oat metabolome discovery phenotypes}

130 Whole metabolome phenotypes were measured from mature seeds using untargeted LC-MS and

131 GC-MS in a diverse oat germplasm panel of 375 inbred lines. These phenotypes have been

132 previously described (Brzozowski et al., 2021; Campbell et al., 2021b; a; Hu et al., 2021). For

133 each metabolite phenotype, measured as relative signal intensity, deregressed best linear

134 unbiased predictors (drBLUPs) could be calculated for 1067 of the LC-MS and 601 of the GC-

135 MS signals as in (Campbell et al., 2021b).

136 We characterized the metabolites by information provided by the Proteomics and

137 Metabolomics Facility at Colorado State University (Fort Collins, CO, USA) (Table 1). The

138 metabolites were annotated by comparison to an in-house spectral library RAMSearch

139 (Broeckling et al., 2016) and MSFinder (Tsugawa et al., 2016), and details of measurement and

140 annotation of this dataset are provided in (Brzozowski et al., 2021; Campbell et al., 2021b; a; Hu

141 et al., 2021). To further characterize the metabolites, we examined the continuous variables of

142 retention time (a measure of polarity, where, using a reverse phase column, a lower retention

143 time indicates greater polarity), molecular mass, and genomic heritability (de los Campos et al.,

144 2015). We also used the provided categorical variables of instrument type (LC, GC), and

145 multiple levels of metabolite type as identified by ClassyFire (Djoumbou Feunang et al., 2016)

146 by superclass, class and subclass. ClassyFire was run in the ClassyFire Batch Compound

147 Classification web server (https://cfb.fiehnlab.ucdavis.edu/) on July 1, 2021. 
150 All analyses were conducted in the R programming environment (R Core Team, 2016). We

151 obtained genotyping-by-sequencing (GBS) data from T3/Oat (https://oat.triticeaetoolbox.org/)

152 for 342 individuals in a diverse panel of oat genotypes as described in (Campbell et al., 2021b).

153 The GBS data was filtered (less than $40 \%$ missingness, minor allele frequency greater than 0.02 )

154 and imputed with glmnet (Friedman et al., 2010). Of these 73,527 markers, the 54,284 that could

155 be anchored to the genome (PepsiCO OT3098v1;

156 https://wheat.pw.usda.gov/GG3/graingenes_downloads/oat-ot3098-pepsico) were used. A

157 principal component (PC) analysis was conducted using the centered and scaled matrix of allele

158 dosages with the function 'prcomp', and percent variance explained by each PC was found using

159 the 'fviz_eig' function. By examination of the scree plot, the first five PCs (accounting for $21.8 \%$

160 of the variance) were chosen for use in analysis. A kinship matrix was calculated using the

161 'A.mat' function, and genomic heritability was calculated using variance components extracted

162 from the 'kin.blup' function, both in the R package rrBLUP (Endelman, 2011).

163

Genome wide association study in discovery panel

165 A single-trait genome-wide association study was conducted for all metabolites (mGWAS) in the 166 statgenGWAS package (Rossum \& Kruijer, 2020) using the kinship matrix and using five PCs as

167 covariates. A false discovery rate correction was used on $p$-values for each metabolite, and a

168 result was considered significant if $p_{F D R}<0.05$.

169

\section{Defining metabolite kernels from discovery panel}

171 We defined sets of SNPs that may broadly shape the measured seed metabolome ("general"

172 kernels), and those that are more specific to lipids ("lipid" kernels) (Table 2). For general 
173 kernels, we selected SNPs that were significant mGWAS results for: (1) three or more LC or GC

174 metabolites ( "Any3"), (2) at least one LC metabolite and at least one GC metabolite

175 (“LCGC2"), (3) four or more LC metabolites (“LC4”), or (4) two or more GC metabolites

176 (“GC2"). The different criteria used to construct LC4 and GC2 were chosen to compare a similar

177 proportion of metabolites per instrument $(0.37 \%$ and $0.33 \%$, respectively). To determine if these

178 kernels represented more SNPs than expected by chance, we used a Poisson model to determine

179 the probability of observing the same significant SNP for multiple metabolites to the rate of SNP

180 inclusion in a kernel using the 'ppois' function in R.

181 We defined lipid kernels by significant mGWAS results of LC-MS lipids based on a

182 hierarchy of pathway specificity. First, we defined a kernel of significant mGWAS results shared

183 by two or more metabolites classified as 'Lipids and lipid-like molecules' superclass ("Lipid").

184 We also created two terpenoid biosynthesis pathway kernels of significant mGWAS results from

185 metabolites classified as (1) the subset of terpenoids predominantly produced by the Mevalonate

186 Acid pathway ("MVA"; subclasses of 'Triterpenoids' and 'Sesquiterpenoids'), and (2) the subset

187 of terpenoids predominantly produced by the Methylerythritol Phosphate pathway ("MEP”;

188 subclasses of 'Diterpenoids' and 'Tetraterpenoids'). Again, criteria for including SNPs were

189 modified by kernel to create kernels of similar size.

190 We visualized genome location by plotting the number of significant mGWAS results in

191 10Mb bins. For all further analyses we added all other SNPs in strong linkage disequilibrium LD

$192\left(r^{2}>0.5\right)$ to each set of SNPs. We used the most recent transcriptome annotations (Hu et al., 2020)

193 and noted SNPs that were within, or up to $2.5 \mathrm{~kb}$ upstream of genes.

194

195

\section{Descriptive analyses of metabolite kernels}


196 We examined if metabolite characteristics were explanatory for the GWAS results identified.

197 First, we tested if there was a relationship between metabolite heritability and retention time,

198 molecular mass, or metabolite superclass. For retention time and molecular mass, we used a

199 linear model with the ' $1 \mathrm{~m}$ ' function with heritability as the response variable, and tested effect

200 significance by ANOVA. We also calculated mean heritability for metabolites by ClassyFire 201 superclass.

202 We tested if focal superclass categories were enriched or depleted in each of the kernels

203 using the 'phyper' function in R. We also calculated the mean Euclidean distance between

204 metabolites in the kernels, using a matrix with metabolites in rows and oat lines in columns and

205 the cells containing their scaled and centered drBLUPs with the 'dist' function with the

206 'euclidean' method in R. To compare distance between metabolites contributing to the kernel to

207 metabolites not contributing to the kernel, we used the Mann-Whitney $U$ test implemented with

208 the 'wilcox.test' function in R.

\section{Oat metabolome validation phenotypes}

211 We used a validation germplasm panel to test the transferability of kernels between populations.

212 This population is described by (Brzozowski et al., 2021). Briefly, a panel of 235 inbred lines

213 was evaluated in three Midwest production environments (Minnesota, "MN"; South Dakota,

214 "SD" and Wisconsin, "WI”). For this analysis, we removed lines that overlapped with our

215 discovery (diverse) panel, leaving 212 lines in MN and SD and 208 lines in WI. The relationship

216 between the discovery and validation panels are described in (Hu et al., 2021), named as

217 'discovery' and 'elite’ panels, respectively. 
Deregressed BLUP (drBLUP) were calculated as in (Campbell et al., 2021b) where data

219 was cube-root transformed, and there were 397 LC and 243 GC metabolites (640 total) for which

220 drBLUPs could be calculated. Metabolite heritability and percent variation described by kernels

221 were calculated as above. Spearman's rank correlation of metabolite heritability across

222 environments was evaluated with the 'cor.test' function in R. In addition to examining the

223 metabolome as a whole, we also evaluated outcomes for the specialized metabolites,

224 avenanthramides, avenacins and avenacosides as described in (Brzozowski et al., 2021).

225 Metabolite drBLUPs and annotations are provided as Supporting Data.

228 We conducted genomic prediction for metabolites $(n=640)$ and genotypes $(n=189)$ measured in

229 the validation panel separately in all environments. We then fit a two-kernel GBLUP model

230 using the selected SNPs to construct Gaussian kernels as described in (de Los Campos, 2018)

231 and (Cuevas et al., 2020) in the R package 'BGLR' (Pérez \& de los Campos, 2014) with 20000

232 iterations and a burn in of 5000. We conducted five-fold cross validation with 50 replicates,

233 where folds were consistent between metabolites and environments, and report the correlation $(r)$

234 between predicted and observed values.

\section{Evaluation of genomic prediction results in the validation panel}

237 We evaluated if the two kernel metabolite models had significantly higher or lower prediction 238 accuracies than GBLUP. First, we used paired one-sided Wilcoxon rank-sum tests using the 239 mean prediction accuracy per metabolite and model. We also tested if mean prediction accuracy

240 varied between environments using a Kruskal-Wallis test. Finally, we conducted paired tests 
241 between the two-kernel metabolite models and GBLUP by models and metabolites using

242 accuracy of each of fifty replicates to understand which were significantly different from

243 GBLUP. In both cases, we report significant results as $p_{B O N F}<0.05$.

244 We partitioned genetic variation from the two kernels (metabolite kernel, rest-of-genome

245 kernel) to assess the percent variation that was explained by the metabolite kernels. We

246 compared the metabolite kernels described above to kernels constructed from random draws of

247 loci with significant mGWAS results that were not included in metabolite kernels $(n=4238$

248 SNPs). We had 10 random draws of 20, 50, 100, 500, 900 and 1800 SNPs, and added SNPs in

249 LD as above to span the size range of kernels (Table S3). The genetic variation explained by

250 these null kernels relative to metabolite kernels was evaluated as well as the impact of kernel

251 size on genetic variation explained.

252 To examine differences between environments, we created matrices of prediction

253 accuracies with models in rows and each metabolite in columns by environment. We then

254 calculated the distance between models (by metabolites of each instrument) and performed

255 hierarchical clustering within an environment and compared groupings of models.

256 Finally, we tested if similar metabolites have similar model ranks, measured by

257 Spearman's rank correlation. We defined 'similar' in three ways. First, we examined results for

258 seven specialized metabolites important for human health, or plant resistance to disease for

259 which we have high-confidence annotations: the avenanthramides, avenacins and avenacosides

260 (Brzozowski et al., 2021). Second, we used finer scale structural descriptions ('Class'

261 description) of metabolites of the 'Lipid and Lipid-like compounds' ClassyFire Superclass

262 ( $n=91)$. Third, we attempted an annotation-free method where we computed the mean Euclidean

263 distance between metabolites in the kernels with metabolites in rows and oat lines in columns 
264 and the cells containing their scaled and centered drBLUPs with the 'dist' function with the

265 'euclidean' method in R. We then performed hierarchical clustering to define 10 groups of

266 metabolites for each of the environments using the 'hclust' function both in $\mathrm{R}$.

\section{RESULTS}

\section{Oat seed metabolome of the discovery panel}

270 Using untargeted metabolomics, we detected 1067 LC-MS and 601 GC-MS metabolites for

271 which deregressed BLUPs could be calculated, and characterized the metabolites by chemical

272 properties as well as retention time and molecular mass. The LC-MS metabolites had greater

273 genomic heritability (mean, $h^{2}=0.23$ ) than GC-MS metabolites (mean, $h^{2}=0.13$ ) (Figure 1a). For

274 both LC-MS and GC-MS metabolites, we found that heritability was greater at lower retention

275 times (greater polarity) and for larger molecular masses, even when the lowest heritability

276 compounds were excluded (Figure S1). The LC-MS metabolites were more densely annotated

277 than the GC-MS metabolites, and lipids were the most common classification (49\%) of LC-MS

278 metabolites (Table 1). While we did not observe any relationship between heritability and

279 metabolite structural characteristics, annotated GC-MS metabolites had higher heritability than

280 unannotated metabolites (Table S1).

281 A metabolite genome-wide association study mGWAS was conducted for all metabolites,

282 and 368 metabolites had at least one significant SNP $\left(p_{F D R}<0.05\right)$ and 8415 unique SNPs

283 (15.5\% of total SNPs) were implicated. Of these, there were 282 LC-MS (5728 unique SNPs,

$28410.6 \%$ of total SNPs), and 86 GC-MS (3544 unique SNPs, 6.5\% of total SNPs) metabolites with

285 a significant association. The metabolites with significant associations tended to have higher

286 heritability than those without for both LC-MS and GC-MS metabolites (Figure 1b; Figure S2). 


\section{Defining kernels for whole genome regression}

289 Using the mGWAS results, we defined kernels to capture loci that broadly shape the metabolome

290 ("general"), and loci specific to metabolite structures or pathways. We hypothesized that the

291 general kernels would broadly improve metabolite prediction, while kernels customized to

292 specific lipids would improve prediction of their respective metabolites (Table 2).

294 9-78 metabolites for the general and specific kernels, respectively (Table S2), with some

295 metabolites and SNPs contributing to multiple kernels (Figure S3). Correlations between kernel

296 off-diagonal elements ranged from $r=0.12-0.83$, and the two kernels relying on mGWAS from

297 GC-MS ('LCGC2' and 'GC2') were the most distinct from other kernels (Figure S4).

In evaluating if kernels were enriched for mGWAS loci from particular metabolites, we

299 found that LC-MS metabolites contributing to metabolite kernels were significantly depleted for

300 lipids (Figure 2). GC-MS metabolites were more sparsely annotated than LC-MS compounds,

301 but metabolites with mGWAS results were enriched for annotated compounds (Figure 2). We

302 also evaluated the pairwise Euclidean distance between metabolites to test in an annotation-free

303 way if more similar metabolites had similar mGWAS results. The GC-MS metabolites

304 contributing to kernels had significantly reduced distance between metabolites compared to all

305 GC-MS metabolites, but there was no reduced distance of LC-MS metabolites contributing to

306 kernels (Figure S5).

307 We compared the rate of SNPs meeting criteria for inclusion in a kernel (e.g., significant

308 mGWAS result shared by three metabolites) to the empirical rate of mGWAS results in this oat

309 population. Compared to a random draw from a Poisson distribution, there were more SNPs 
meeting criteria than expected ('Any3', $\lambda=0.16, p=5.5 \mathrm{e}-04$; 'LCGC2', $\lambda=0.16, p=1.2 \mathrm{e}-04$;

311 'LC4', $\lambda=0.11, p=4.8 \mathrm{e}-06$; ' $\mathrm{GC} 2$ ', $\lambda=0.07, p=0.002)$. The SNPs for the general kernels were

312 identified on most chromosomes but clustered within chromosomes (Figure S6). The lipid-

313 related kernels had the most SNPs on chromosome 5A and 5C (Figure S7). Finally, kernels had

314 a range of gene density, with a maximum $11 \%$ of SNPs in the 'MVA' kernel being in a gene and 315 a minimum of $6.7 \%$ in 'LCGC2' (Table 3).

\section{Oat seed metabolome of the validation panel}

318 We tested if kernels developed in the discovery panel improved prediction accuracy for 319 metabolites in a validation panel evaluated in three environments (Minnesota, "MN"; South 320 Dakota, "SD” and Wisconsin, "WI”) that had 397 LC-MS and 243 GC-MS metabolites.

321 Although the measurements do not allow for direct comparison of all individual metabolites to

322 those in the discovery panel (due to currently no robust method to map all untargeted metabolites

323 from one panel to another and quantify them accurately, Hu et al. 2021), the metabolite

324 classification parameters were consistent across the two panels. Like the discovery panel, LC-

325 MS metabolites had greater mean heritability $\left(h^{2}: \mathrm{MN}=0.30, \mathrm{SD}=0.17, \mathrm{WI}=0.17\right)$ than GC-MS

326 metabolites $\left(h^{2}: \mathrm{MN}=0.10, \mathrm{SD}=0.09, \mathrm{WI}=0.14\right)$ and heritability was positively correlated across

327 environments (Table S4). Metabolite classifications were available for the LC-MS metabolites

328 only, and lipids were the most common annotation (23\%), but there were no trends in heritability

329 by metabolite type (Table S5). Finally, except for LC-MS metabolites in MN, there were

330 significant negative relationships between heritability and retention time (Figure S8, Table S6). 
333 Mean prediction accuracy of two-kernel (metabolite kernel and rest-of-genome kernel) genomic

334 prediction models from five-fold cross validation ranged from 0.24-0.34 for LC-MS and 0.13-

3350.17 for GC-MS metabolites, where prediction accuracy was highest for LC-MS metabolites in

336 MN and lowest for GC-MS metabolites in MN and SD (Table 4). The 'LC4' kernel improved

337 and the 'GC2' kernel reduced prediction accuracy of LC-MS metabolites over GBLUP in all

338 three environments (Figure 3a). The 'Any3' kernel also improved prediction accuracy of LC-

339 MS metabolites over GBLUP in two environments, as did the 'MVA' kernel, contrary to our

340 expectation that the 'MVA' kernel specificity would not result in improved prediction accuracy

341 for a broad range of metabolites (Figure 3a). No kernel improved prediction accuracy of GC-MS

342 metabolites over GBLUP, but the 'LCGC2' kernel decreased accuracy in two environments

343 (Figure 3b).

344 Individual metabolites with higher genomic heritability had greater prediction accuracy

$345\left(\mathrm{R}^{2}\right.$ adj $=0.61-0.79$; Figure $\left.\mathbf{S 9}\right)$. Using paired tests to compare the two kernel metabolite models to

346 GBLUP for each metabolite, the most metabolites (LC-MS and GC-MS) with significant

347 improvements in accuracy were for the 'MVA', 'LC4' and 'Any3' kernels, while the most

348 metabolites with significant reductions in accuracy where for ' $\mathrm{GC} 2$ ' and 'MEP' kernels for LC-

349 MS metabolites, and no clear patterns for GC-MS metabolites (Table 5). On average, 37\% and

$35026 \%$ of LC-MS and GC-MS metabolites, respectively had higher prediction accuracy with any of

351 the two-kernel metabolite models than GBLUP, and $47 \%$ and $28 \%$ had lower prediction

352 accuracy with any of the two-kernel metabolite models than GBLUP. Of all metabolites

353 identified to have significant changes in accuracy compared to GBLUP, two-thirds were unique

354 to one environment (Figure S10). 
Using the metabolite kernel and the rest-of-genome kernel to partition genetic variation,

356

357

358

359

360

361

362

363

364

365

366

367

368

369

370

371

372

373

374

375

376

377

we found that the metabolite kernels consistently accounted for almost half of total heritability

(Figure 4). The 'Any3' and 'LC4' kernels accounted for more percent heritability for LC-MS than GC-MS metabolites in two environments, and the 'GC2' kernel accounted for more percent heritability explained for GC-MS than LC-MS metabolites in all environments (Figure 4a).

Percent heritability explained was generally lower in MN than SD and WI for LC-MS

metabolites (Figure 4b), and there were differences observed between environments for GC-MS metabolites for the 'LCGC2' and 'GC2' kernels (Figure 4c). There were weak negative relationships between metabolite genomic heritability and percent heritability explained by the kernel $\left(\mathrm{R}^{2}\right.$ adj $=0.05-0.15$; Figure S11), but no relationship between percent heritability explained by the kernel with kernel size (Table S7).

We compared genetic variation attributed to metabolite kernels to random kernels of similar sizes, constructed from SNPs that were significant $\left(p_{F D R}<0.05\right)$ mGWAS results that were not included in kernels, and found that, for LC-MS metabolites, the 'Any3', 'LC4', 'Lipid' and 'MVA' kernels explained more genetic variance but the 'LCGC2' and 'GC2' kernels explained less (Table S7). In contrast, metabolite kernels never explained more percent genetic variation than random mGWAS kernels for GC-MS metabolites (Table S7).

To better understand the effect of the environment on relative model outcomes, we calculated the rank correlation of metabolite prediction accuracy between models and performed hierarchical clustering of the Euclidean distance between ranks. For all metabolites, the 'Any3' and 'LC4' and the 'LCGC2' and 'GC2' kernels grouped in all environments (Figure 5). 
378 We evaluated if similar metabolites had similar model rankings, where we defined metabolite

379 similarity by: (1) known annotations, (2) structural characteristics as classified by ClassyFire,

380 and (3) Euclidean distance between phenotypes.

For seven oat specialized metabolites where high-confidence named annotations are

382 available (avenanthramides, avenacins, avenacosides), there were 24 instances (of the 147 trait,

383 model and environment combinations) where including a metabolite kernel significantly changed

384 prediction accuracy compared to GBLUP (Table S8). We found that similar metabolites had

385 similar ranks of kernels by prediction accuracy in two environments (MN, WI) (Figure 6). These

386 results indicate that when we have access to high-confidence named annotations to define similar

387 metabolites, the similar metabolites have similar prediction results.

We assessed LC-MS metabolites structurally classified as lipids ( $n=91)$, and particularly

389 prediction accuracy of the 'Lipid' compared to others. While the 'Lipid' two-kernel model

390 significantly outperformed GBLUP in only one environment (SD), it generally had higher

391 prediction accuracy than most other kernels besides 'MVA' in two environments (MN, SD)

392 (Figure 7). Other kernels accounted for more heritability than the lipid kernel in only two

393 instances (Figure 7). We defined lipids as 'similar' by 'Class' descriptor (e.g. steroids, or fatty

394 acyls), and anticipated similar model rankings by lipid class. We found lipid Class was not

395 predictive of the model rank (Figure 8), suggesting that structural classifications may not

396 provide effective metabolite groupings.

Finally, without using annotations, we computed the distance between metabolites and

398 performed hierarchical clustering to define 10 metabolite groups per environment. Most of the

399 groups had significantly higher correlations of model rank within group compared to metabolites

400 out of the group (Figure 9). We found that the groups were largely defined by retention time. 
401 Groups with strong within-group correlation had smaller coefficients of variation in retention

402 time $(\mathrm{CV}<20)$ than other groups, but the trends in genomic heritability were not consistent

403 between groups (Table 6). These groups also had less variation in retention time than the lipid

404 Classes (CV> 20; Table S9).

\section{DISCUSSION}

407 Our work tests generalizable frameworks for genomic prediction of a diverse array of plant 408 metabolites. Using a discovery germplasm panel, we identified loci by mGWAS that represent

409 different biological bases - loci that affect multiple types of metabolites to metabolites from

410 specific biochemical pathways. Building kernels from significant mGWAS loci that affect

411 multiple LC-MS metabolites and specific pathways thereof increased prediction accuracy over

412 GBLUP in a validation panel for LC-MS metabolites. No model tested improved prediction of

413 GC-MS metabolites over GBLUP, and kernels from GC-MS metabolites reduced prediction

414 accuracy in some cases. mGWAS-defined kernels accounted for $\sim 45 \%$ of genetic variation, and

415 rank of kernel performance was consistent between environments. An ongoing challenge in

416 developing generalized genomic prediction frameworks is defining metabolite 'similarity'. We

417 found that grouping metabolites by high-confidence named annotations and computationally

418 derived groupings (without annotations) had similar outcomes from the models tested, while

419 metabolites delineated by structural features alone did not. Overall, this work builds from efforts

420 to predict tens of biochemically similar metabolites to metabolome-wide genomic prediction. 
423 We evaluated over 2000 metabolites measured by LC-MS or GC-MS in mature oat seed and

424 found that, on average, metabolites had low to moderate genomic heritability (mean $h^{2}=0.09$ to

425 0.30), with LC-MS metabolites being more heritable than GC-MS metabolites. Other analyses of

426 untargeted metabolites ( $n=900-7000$ metabolites) report wide ranges of broad-sense (not

427 genomic) heritability $\left(\mathrm{H}^{2}\right)$, from a uniform distribution (Zhou et al., 2019), to right (Zhu et al.,

428 2018) and left (Chen et al., 2016) skews. While some differences in heritability between studies

429 could be attributed to the tissue and developmental specificity of metabolites (Soltis \&

430 Kliebenstein, 2015), we also found that metabolite heritability covaries with column retention

431 time (related to metabolite polarity). While retention time was not evaluated, (Zhou et al., 2019)

432 found that less common features tended to have lower heritability that they attributed to machine

433 artifact. This suggests that parameters such as specific extraction (e.g., if the extracting solvent

434 more efficiently extracts polar or non-polar compounds), or signal processing methods may

435 affect error variation.

436 By conducting mGWAS for the 1668 metabolites in the discovery panel, we found that a

437 greater proportion of LC-MS than GC-MS metabolites had significant mGWAS results, even

438 when controlling for heritability differences, suggesting that more LC-MS metabolites have an

439 oligogenic genetic architecture. Overall, primary metabolites (measured by GC-MS) tend to be

440 dominantly inherited (Schauer et al., 2008; Fernie \& Tohge, 2017), and variation is determined

441 by multiple small effect loci (Soltis \& Kliebenstein, 2015). In contrast, specialized metabolites

442 (measured by LC-MS) generally arise from variation in primary metabolism (Moghe \& Last,

443 2015; Maeda, 2019) including enzyme neofunctionalization (Pichersky \& Gang, 2000; Fernie \&

444 Tohge, 2017). Nonetheless, selection type (e.g., direction or stabilizing) in crops is more

445 important in predicting loci effects than type of metabolite per se (Soltis \& Kliebenstein, 2015). 
446 There are multiple examples of balancing selection for metabolite concentration (e.g., as

447 defensive metabolite, or regionally preferred crop aesthetic or flavor) (Soltis \& Kliebenstein,

448 2015), and (Campbell et al., 2021b) proposed that optimizing or stabilizing selection pressures

449 predominately shape the oat seed metabolome.

450 Another factor that may contribute to the differences between the mGWAS results for

451 GC-MS (primary) and LC-MS (specialized) metabolites is that metabolites were measured in

452 mature seed. Primary metabolites decreased in Arabidopsis seed during reserve accumulation,

453 but then increased during seed desiccation (putatively for availability for germination energy)

454 (Fait et al., 2006). In contrast, primary metabolites in rice consistently decrease beginning at

455 desiccation (Hu et al., 2016). In a time-series transcriptome-wide analysis of developing oat

456 seed, expressed genes had enriched GO terms for photosynthesis until 23 days after anthesis

457 (DAA), followed by an enrichment of GO terms for nutrient reservoir activity beginning at 28

458 DAA (Hu et al., 2020). These results suggest that the metabolomic dynamics in developing oat

459 seed may be similar to those of rice, and point to a need for multiple metabolome measures

460 during seed development.

\section{Potential for generalizable approaches for genomic prediction of metabolites}

463 We used multiple criteria for constructing metabolite kernels to test hypotheses of which

464 biological partition may be the most enriched for causal SNPs. We developed kernels to

465 encompass general metabolome-wide information from both or single LC-MS and GC-MS

466 instruments ('Any3', 'LCGC2', and LC4', 'GC2', respectively), or metabolites structurally

467 identified as lipids ('Lipid'), and pathways thereof ('MVA', 'MEP') for two-kernel genomic

468 prediction. Importantly, to make our results relevant for plant breeding programs, we selected 
469

470

471

472

473

474

475

476

477

478

479

480

481

482

483

484

485

486

487

SNPs from a diverse 'discovery' panel, and evaluated prediction accuracy in another more elite population evaluated in multiple environments.

Metabolite kernels accounted for a high percent of trait genetic variation, and the 'Any3', 'LC4', and 'MVA' kernels consistently increased prediction accuracy over GBLUP for LC-MS metabolites. While the 'MVA' kernels included the highest number of SNPs in genes of any of the kernels, high gene richness did not always translate to high prediction accuracy (e.g., the 'Lipid' kernel), indicating that gene richness alone does not account for our results.

The general kernels likely include loci that affect multiple metabolites, as loci with pleiotropy and epistatic interactions are common for metabolites (Soltis \& Kliebenstein, 2015), and we hypothesized that using these kernels would increase prediction accuracy of the most metabolites. The 'Any3' and 'LC4' kernels improved prediction accuracy, and the 'LC4' kernel more so, where the 'LC4' kernel is a subset of the 'Any3' kernel. Our approach can be compared to factor analysis recently used in genomic prediction of several oat fatty acids (Campbell et al., 2021b). In both cases (results from individual mGWAS and result from GWAS of factors), multi-kernel models improved prediction accuracy. Nonetheless, many factors extracted from oat metabolomic data were enriched for lipids (Campbell et al., 2021b), while our 'Any3' and 'LC4' kernels were depleted for lipids, indicating that we are capturing different information than the factor analysis. Overall, these results suggest that distilling results from the entire metabolome identifies SNPs that affect multiple metabolites and improves prediction accuracy.

8
Contrary to our expectations, the 'MVA' kernel that incorporated only a specific branch of terpenoid biosynthesis (e.g., triterpenoids and sesquiterpenoids) improved prediction accuracy of LC-MS metabolites metabolome-wide as much as the general 'LC4' and 'Any3' kernels. While the 'MEP' kernel representing another terpenoid biosynthetic pathway (e.g., diterpenoids 
492 and carotenoids) did not improve accuracy, these pathways function largely independently, and

493 sometimes antagonistically (Rodríguez-Concepción \& Boronat, 2015). Increased prediction

494 accuracy from the 'MVA' kernel suggests that loci governing variation in specific pathways may

495 translate across populations for metabolome-wide prediction. Alternatively, this result could be

496 specific to terpenoids: (Turner-Hissong et al., 2020) reported that a terpenoid gene kernel

497 improved prediction of a free amino acid, isoleucine, in Arabidopsis seed where the terpenoids

498 are unrelated to isoleucine biosynthesis. It would be intriguing to test if terpenoid-related kernels

499 improve prediction accuracy of seemingly unrelated metabolites in other non-seed tissues (with

500 lower oil content) to assess if energetic tradeoffs are responsible for this observation.

A kernel derived from mGWAS results from LC-MS metabolites structurally identified

502 as lipids ('Lipid') in the discovery panel, did not improve prediction accuracy metabolome-wide,

503 or for lipids over GBLUP in the validation panel. (Campbell et al., 2021b) found that latent

504 factors that were enriched for lipids did not significantly improve prediction accuracy of

505 proteins, likely due to high negative genetic correlation between those traits and that factor

506 loadings included more metabolites than just lipids. The 'Lipid' kernel here was also potentially

507 too expansive of a categorization and may have led to kernels containing genomic regions with

508 shared regulation but opposing effects. This result suggests that grouping metabolites by shared

509 regulatory control may be more beneficial (e.g., 'MVA'), and will become more feasible with

510 improved genomic resources.

511 Finally, no method we tested improved prediction accuracy of GC-MS metabolites, and

512 kernels from solely mGWAS results from GC-MS metabolites ('GC2') reduced prediction

513 accuracy of LC-MS metabolites. This may be because GC-MS metabolites had lower heritability

514 (potentially due to lower phenotypic variation in mature seed, constraints on potential genetic 
515 variation), fewer mGWAS results, and thus provided less reliable information. Overall, these

516 results highlight that combining multiple metabolomics datasets from different instruments may

517 have limited efficacy, depending on, for instance, development stage sampled.

\section{Strategies for categorizing 'similar' metabolites}

520 In building generalized frameworks, it would be useful to have high-throughput methods for

521 identifying similar metabolites to which to apply the same prediction method. A key challenge,

522 however, is how 'similar' is defined. We tested three definitions of 'similar': high-confidence

523 named annotations of known metabolites (difficult to obtain, high biological information),

524 automated metabolite classification by chemical structure (moderate effort to obtain, some

525 biological information), and by an annotation-free measure of similarity (easy to obtain, no

526 biological information). Overall, groups of metabolites by named annotations and by the

527 annotation-free measure, had consistent ranks of the models tested. In the annotation-free

528 grouping, we found that retention time was an important predictor of group association. As

529 metabolite annotations provide useful biological information, we look forward to more high

530 confidence annotations as databases continue to grow (Afendi et al., 2012).

531 Defining 'similar' by structural classification was the least successful method, perhaps

532 because structural classifications do not broadly correspond to a biosynthetic pathway

533 (Djoumbou Feunang et al., 2016). A caveat in examining relative model rankings is that we did

534 not specifically design kernels to evenly represent the space of all potential kernels but, as the

535 purpose of this study was to test different biological rationales, this analysis is informative for

536 understanding differences between approaches. 


\section{CONCLUSIONS}

539 We are building towards a generalized framework for genomic prediction of metabolites by

540 investigating how we can efficiently extract information from metabolomics data, integrate

541 biology to find the most informative loci, and then test for which metabolites these strategies are

542 most successful. Our work extends the foundational metabolomics work done in model

543 organisms like Arabidopsis, tomato, maize (Fernie \& Tohge, 2017) and on conserved

544 biochemical pathways (Wager \& Li, 2018), to provide strategies for genomic prediction of

545 multiple, diverse metabolites in non-model crops. Overall, we show that integrating whole

546 metabolome or specific pathway information improves genomic prediction accuracy and

547 translates across populations within a species. This work also provides a framework for testing

548 such models between closely related species by transfer learning (Wang et al., 2020). 


\section{ACKNOWLEDGEMENTS}

550 Corey D. Broeckling conducted the metabolomics extraction, measurement, and data processing

551 at the Bioanalysis and Omics Center of the Analytical Resources Core at Colorado State

552 University (Fort Collins, CO USA). Funding for this research was provided by the United States

553 Department of Agriculture - National Institute of Food and Agriculture - Agriculture and Food

554 Research Initiative (USDA NIFA-AFRI) grant (2017-67007-26502), and the USDA Agricultural

555 Research Service Project Number 8062-21000-045-000-D.

\section{CONFLICT OF INTEREST}

558 The authors declare no conflict of interest.

\section{AUTHOR CONTRIBUTIONS}

561 JLJ, MAG and MES designed the research. LJB, HH, and MTC analyzed the data, and HH,

562 MTC, MC, LG, KPS and MES conducted experiments. LJB, MAG and JLJ wrote the manuscript 563 and all co-authors were involved in editing the manuscript.

\section{DATA AVAILABILITY}

566 Deregressed BLUPs of the metabolites for the discovery (diverse) germplasm panel are available

567 in the supplementary material of (Campbell et al., 2021b). Deregressed BLUPs of the

568 metabolites for the validation germplasm panel is provided as Supporting File 1. Genotype data

569 is as used in (Brzozowski et al., 2021) and available at

570 https://datacommons.cyverse.org/browse/iplant/home/shared/GoreLab/dataFromPubs/Brzozowsk 
bioRxiv preprint doi: https://doi.org/10.1101/2021.11.24.469870; this version posted November 25, 2021. The copyright holder for this preprint (which was not certified by peer review) is the author/funder. All rights reserved. No reuse allowed without permission.

571 i_OatMetabolome_2021. The R code for these analyses is available on a public repository in

572 https://github.com/ljbrzozowski/OatMetaboliteGenomicPrediction

573 


\section{SUPPORTING INFORMATION CONTENTS}

575 File S1. Validation germplasm panel metabolite information

576 Figure S1. Linear regressions of metabolite heritability by retention time and molecular mass.

577 Figure S2. Number of metabolites with significant GWAS results by instrument type.

578 Figure S3. The number of metabolites and SNPs contributing to kernels.

579 Figure S4. Correlation between off-diagonal elements in metabolite kernels.

580 Figure S5. Mean Euclidean distance between metabolites that contribute to metabolite kernels.

581 Figure S6. Genomic distribution of metabolite GWAS results and the general kernels.

582 Figure S7. Genomic distribution of metabolite GWAS results and the lipid kernels.

583 Figure S8. Validation panel metabolite genomic heritability and relationship to retention time.

584 Figure S9. Mean cross-fold validation accuracy of metabolites compared to genomic heritability

585 Figure S10. Metabolites where any two-kernel metabolite model improved or reduced genomic

586 prediction accuracy over GBLUP.

587 Figure S11. Percent genetic variation attributed to the metabolite kernel compared to metabolite 588 genomic heritability.

589 Table S1. Mean metabolite heritability by ClassyFire superclass groups for the discovery panel.

590 Table S2. Number of metabolites in each metabolite kernel.

591 Table S3. Number of SNPs in each metabolite kernel.

592 Table S4. Spearman's rank correlation of metabolite heritability across validation panel

593 environments.

594 Table S5. Validation panel LC-MS metabolite ClassyFire 'Superclass' count and heritability.

595 Table S6. Validation panel linear regression between retention time and heritability 
596 Table S7. Comparison of percent genetic variance (percent heritability) by metabolite kernels to

597 mGWAS random kernels by instrument, model and environment.

598 Table S8. Significant differences in prediction accuracy compared to GBLUP for seven

599 specialized metabolites.

600 Table S9. Coefficient of variation in retention time of LC-MS lipids by Class. 


\section{REFERENCES}

Afendi, F.M., Okada, T., Yamazaki, M., Hirai-Morita, A., Nakamura, Y., Nakamura, K., Ikeda, S., Takahashi, H., Altaf-Ul-Amin, M., Darusman, L.K., Saito, K., \& Kanaya, S. (2012). KNApSAcK family databases: Integrated metabolite-plant species databases for multifaceted plant research. Plant and Cell Physiology, 53, 1-12. https://doi.org/10.1093/pcp/pcr165

Baseggio, M., Murray, M., Magallanes-Lundback, M., Kaczmar, N., Chamness, J., Buckler, E.S., Smith, M.E., DellaPenna, D., Tracy, W.F., \& Gore, M.A. (2019). Genome-Wide Association and Genomic Prediction Models of Tocochromanols in Fresh Sweet Corn Kernels. The Plant Genome, 12, 180038. https://doi.org/10.3835/plantgenome2018.06.0038

Broeckling, C.D., Ganna, A., Layer, M., Brown, K., Sutton, B., Ingelsson, E., Peers, G., \& Prenni, J.E. (2016). Enabling Efficient and Confident Annotation of LC-MS Metabolomics Data through MS1 Spectrum and Time Prediction. Analytical Chemistry, 88, 9226-9234. https://doi.org/10.1021/acs.analchem.6b02479

Brzozowski, L.J., Hu, H., Campbell, M.T., Broeckling, C.D., Caffe-Treml, M., Gutiérrez, L., Smith, K.P., Sorrells, M.E., Gore, M.A., \& Jannink, J.-L. (2021). Selection for seed size has indirectly shaped specialized metabolite abundance in oat (Avena sativa L.). BioRvix, . https://doi.org/10.1101/2021.08.18.454785

Campbell, M.T., Hu, H., Yeats, T.H., Brzozowski, L.J., Caffe-Treml, M., Gutiérrez, L., Smith, K.P., Sorrells, M.E., Gore, M.A., \& Jannink, J.-L. (2021)(a). Improving Genomic Prediction for Seed Quality Traits in Oat (Avena sativa L.) Using Trait-Specific Relationship Matrices. Frontiers in Genetics, 12, 643733. https://doi.org/10.3389/fgene.2021.643733

Campbell, M.T., Hu, H., Yeats, T.H., Caffe-Treml, M., Gutiérrez, L., Smith, K.P., Sorrells, M.E., Gore, M.A., \& Jannink, J.-L. (2021)(b). Translating insights from the seed metabolome into improved prediction for lipid-composition traits in oat (Avena sativa L.). Genetics, 217, iyaa043. https://doi.org/10.1093/genetics/iyaa043

de los Campos, G., Sorensen, D., \& Gianola, D. (2015). Genomic Heritability: What Is It?. PLOS Genetics, 11, e1005048. https://doi.org/10.1371/journal.pgen.1005048

Carlson, M.O., Montilla-Bascon, G., Hoekenga, O.A., Tinker, N.A., Poland, J., Baseggio, M., Sorrells, M.E., Jannink, J.L., Gore, M.A., \& Yeats, T.H. (2019). Multivariate genome-wide association analyses reveal the genetic basis of seed fatty acid composition in oat (Avena sativa L.). G3: Genes, Genomes, Genetics, 9, 2963-2975. https://doi.org/10.1534/g3.119.400228

Chen, W., Wang, W., Peng, M., Gong, L., Gao, Y., Wan, J., Wang, S., Shi, L., Zhou, B., Li, Z., Peng, X., Yang, C., Qu, L., Liu, X., \& Luo, J. (2016). Comparative and parallel genome-wide association studies for metabolic and agronomic traits in cereals. Nature Communications, 7, 12767. https://doi.org/10.1038/ncomms12767

Cuevas, J., Montesinos-López, O.A., Martini, J.W.R., Pérez-Rodríguez, P., Lillemo, M., \& Crossa, J. (2020). Approximate Genome-Based Kernel Models for Large Data Sets Including Main Effects and Interactions. Frontiers in Genetics, 11, 567757. https://doi.org/10.3389/fgene.2020.567757

Diepenbrock, C.H., Ilut, D.C., Magallanes-Lundback, M., Kandianis, C.B., Lipka, A.E., Bradbury, P.J., Holland, J.B., Hamilton, J.P., Wooldridge, E., Vaillancourt, B., Góngora-Castillo, E., Wallace, J.G., Cepela, J., Mateos-Hernandez, M., Owens, B.F., Tiede, T., Buckler, E.S., Rocheford, T., 
Buell, C.R., Gore, M.A., \& DellaPenna, D. (2021). Eleven biosynthetic genes explain the majority of natural variation in carotenoid levels in maize grain. The Plant Cell, 33, 882-900. https://doi.org/10.1093/plcell/koab032

Diepenbrock, C.H., Kandianis, C.B., Lipka, A.E., Magallanes-Lundback, M., Vaillancourt, B., GóngoraCastillo, E., Wallace, J.G., Cepela, J., Mesberg, A., Bradbury, P.J., Ilut, D.C., Mateos-Hernandez, M., Hamilton, J., Owens, B.F., Tiede, T., Buckler, E.S., Rocheford, T., Buell, C.R., Gore, M.A., \& DellaPenna, D. (2017). Novel Loci Underlie Natural Variation in Vitamin E Levels in Maize Grain. The Plant Cell, 29, 2374-2392. https://doi.org/10.1105/tpc.17.00475

Djoumbou Feunang, Y., Eisner, R., Knox, C., Chepelev, L., Hastings, J., Owen, G., Fahy, E., Steinbeck, C., Subramanian, S., Bolton, E., Greiner, R., \& Wishart, D.S. (2016). ClassyFire: automated chemical classification with a comprehensive, computable taxonomy. Journal of Cheminformatics, 8, 1-20. https://doi.org/10.1186/s13321-016-0174-y

Endelman, J.B. (2011). Ridge Regression and Other Kernels for Genomic Selection with R Package rrBLUP. The Plant Genome, 4, 250-255. https://doi.org/10.3835/plantgenome2011.08.0024

Fait, A., Angelovici, R., Less, H., Ohad, I., Urbanczyk-Wochniak, E., Fernie, A.R., \& Galili, G. (2006). Arabidopsis Seed Development and Germination Is Associated with Temporally Distinct Metabolic Switches. Plant Physiology, 142, 839-854. https://doi.org/10.1104/pp.106.086694

Fernie, A.R., \& Tohge, T. (2017). The Genetics of Plant Metabolism. Annual Review of Genetics, 51, $287-310$

Friedman, J., Hastie, T., \& Tibshirani, R. (2010). Regularization Paths for Generalized Linear Models via Coordinate DescentJournal of. Journal of Statistical Software, 33, 1-22

Heffner, E.L., Sorrells, M.E., \& Jannink, J.L. (2009). Genomic selection for crop improvement. Crop Science, 49, 1-12. https://doi.org/10.2135/cropsci2008.08.0512

Hernandez, C., Wyatt, L.E., \& Mazourek, M. (2020). Genomic Prediction and Selection for Fruit Traits in Winter Squash. G3: Genes, Genomes, Genetics, 10, 3601-3610

Heslot, N., Jannink, J.-L., \& Sorrells, M.E. (2015). Perspectives for Genomic Selection Applications and Research in Plants. Crop Science, 55, 1-12. https://doi.org/10.2135/cropsci2014.03.0249

Hu, C., Tohge, T., Chan, S.-A., Song, Y., Rao, J., Cui, B., Lin, H., Wang, L., Fernie, A.R., Zhang, D., \& Shi, J. (2016). Identification of Conserved and Diverse Metabolic Shifts during Rice Grain Development. Scientific Reports, 6, 20942. https://doi.org/10.1038/srep20942

Hu, H., Campbell, M.T., Yeats, T.H., Zheng, X., Runcie, D.E., Covarrubias-Pazaran, G., Broeckling, C., Yao, L., Caffe-Treml, M., Gutiérrez, L., Smith, K.P., Tanaka, J., Hoekenga, O.A., Sorrells, M.E., Gore, M.A., \& Jannink, J.-L. (2021). Multi-omics prediction of oat agronomic and seed nutritional traits across environments and in distantly related populations. Theoretical and Applied Genetics, In press. https://doi.org/10.1101/2021.05.03.442386

Hu, H., Gutierrez-Gonzalez, J.J., Liu, X., Yeats, T.H., Garvin, D.F., Hoekenga, O.A., Sorrells, M.E., Gore, M.A., \& Jannink, J.L. (2020). Heritable temporal gene expression patterns correlate with metabolomic seed content in developing hexaploid oat seed. Plant Biotechnology Journal, 18, 1211-1222. https://doi.org/10.1111/pbi.13286 
681

682

683

684

685

686

687

688

689

690

691

692

693

694

695

696

697

698

699

700

701

702

703

704

705

706

707

708

709

710

711

712

713

714

715

716

Kumar, R., Bohra, A., Pandey, A.K., Pandey, M.K., \& Kumar, A. (2017). Metabolomics for Plant Improvement: Status and Prospects. Frontiers in Plant Science, 8, 1302. https://doi.org/10.3389/fpls.2017.01302

de Los Campos, G. (2018). Various Ways of fitting a “GBLUP” model using BGLR. GitHub,

Luo, J. (2015). Metabolite-based genome-wide association studies in plants. Current Opinion in Plant Biology, 24, 31-38. https://doi.org/10.1016/j.pbi.2015.01.006

Maeda, H.A. (2019). Evolutionary diversification of primary metabolism and its contribution to plant chemical diversity. Frontiers in Plant Science, 10, 1-8. https://doi.org/10.3389/fpls.2019.00881

Moghe, G., \& Last, R.L. (2015). Something old, something new: Conserved enzymes and the evolution of novelty in plant specialized metabolism. Plant Physiology, 169, 1512-1523. https://doi.org/10.1104/pp.15.00994

Owens, B.F., Lipka, A.E., Magallanes-Lundback, M., Tiede, T., Diepenbrock, C.H., Kandianis, C.B., Kim, E., Cepela, J., Mateos-Hernandez, M., Buell, C.R., Buckler, E.S., DellaPenna, D., Gore, M.A., \& Rocheford, T. (2014). A Foundation for Provitamin A Biofortification of Maize: Genome-Wide Association and Genomic Prediction Models of Carotenoid Levels. Genetics, 198, 1699-1716. https://doi.org/10.1534/genetics.114.169979

Pérez, P., \& de los Campos, G. (2014). Genome-Wide Regression and Prediction with the BGLR Statistical Package. Genetics, 198, 483-495. https://doi.org/10.1534/genetics.114.164442

Pichersky, E., \& Gang, D.R. (2000). Genetics and biochemistry of secondary metabolites in plants: an evolutionary perspective. Trends in Plant Science, 5, 439-445. https://doi.org/10.1016/S13601385(00)01741-6

R Core Team. (2016). R: A Language and Environment for Statistical Computing. R Foundation for Statistical Computing, Vienna, Austria.

Rodríguez-Concepción, M., \& Boronat, A. (2015). Breaking new ground in the regulation of the early steps of plant isoprenoid biosynthesis. Current Opinion in Plant Biology, 25, 17-22. https://doi.org/10.1016/j.pbi.2015.04.001

Rossum, B.-J. van, \& Kruijer, W. (2020). Package 'StatgenGWAS. ' CRAN.

Sarup, P., Jensen, J., Ortersen, T., Henryon, M., \& Sorensen, P. (2016). Increased prediction accuracy using a genomic feature model including prior information on quantitative trait locus regions in purebred Danish Duroc pigs. BMC Genomics, 17, 1-16

Schauer, N., Semel, Y., Balbo, I., Steinfath, M., Repsilber, D., Selbig, J., Pleban, T., Zamir, D., \& Fernie, A.R. (2008). Mode of Inheritance of Primary Metabolic Traits in Tomato. The Plant Cell, 20, 509-523. https://doi.org/10.1105/tpc.107.056523

Soltis, N.E., \& Kliebenstein, D.J. (2015). Natural variation of plant metabolism: Genetic mechanisms, interpretive caveats, and evolutionary and mechanistic insights. Plant Physiology, 169, 14561468. https://doi.org/10.1104/pp.15.01108 
Stewart, D., \& McDougall, G. (2014). Oat agriculture, cultivation and breeding targets: Implications for human nutrition and health. British Journal of Nutrition, 112, S50-S57. https://doi.org/10.1017/S0007114514002736

Tsugawa, H., Kind, T., Nakabayashi, R., Yukihira, D., Tanaka, W., Cajka, T., Saito, K., Fiehn, O., \& Arita, M. (2016). Hydrogen Rearrangement Rules: Computational MS/MS Fragmentation and Structure Elucidation Using MS-FINDER Software. Analytical Chemistry, 88, 7946-7958. https://doi.org/10.1021/acs.analchem.6b00770

Turner-Hissong, S.D., Bird, K.A., Lipka, A.E., King, E.G., Beissinger, T.M., \& Angelovici, R. (2020). Genomic Prediction Informed by Biological Processes Expands Our Understanding of the Genetic Architecture Underlying Free Amino Acid Traits in Dry Arabidopsis Seeds. G3 Genes|Genomes|Genetics, 10, 4227-4239. https://doi.org/10.1534/g3.120.401240

Wager, A., \& Li, X. (2018). Exploiting natural variation for accelerating discoveries in plant specialized metabolism. Phytochemistry Reviews, 17, 17-36. https://doi.org/10.1007/s11101-017-9524-2

Wang, H., Cimen, E., Singh, N., \& Buckler, E. (2020). Deep learning for plant genomics and crop improvement. Current Opinion in Plant Biology, 54, 34-41. https://doi.org/10.1016/j.pbi.2019.12.010

Zhou, S., Kremling, K.A., Bandillo, N., Richter, A., Zhang, Y.K., Ahern, K.R., Artyukhin, A.B., Hui, J.X., Younkin, G.C., Schroeder, F.C., Buckler, E.S., \& Jander, G. (2019). Metabolome-scale genome-wide association studies reveal chemical diversity and genetic control of maize specialized metabolites. Plant Cell, 31, 937-955. https://doi.org/10.1105/tpc.18.00772

Zhu, G., Gou, J., Klee, H., \& Huang, S. (2019). Next-Gen Approaches to Flavor-Related Metabolism. Annual Review of Plant Biology, 70, 187-212

Zhu, G., Wang, S., Huang, Z., Zhang, S., Liao, Q., Zhang, C., Lin, T., Qin, M., Peng, M., Yang, C., Cao, X., Han, X., Wang, X., van der Knaap, E., Zhang, Z., Cui, X., Klee, H., Fernie, A.R., Luo, J., \& Huang, S. (2018). Rewiring of the Fruit Metabolome in Tomato Breeding. Cell, 172, 249261.e12. https://doi.org/10.1016/j.cell.2017.12.019 
Figure 1. Genomic heritability (a) all metabolites $(n=1668)$ and (b) metabolites with a significant GWAS ( $n=368)$ result from the discovery panel. The instrument class (LC-MS, or GC-MS) is denoted by color (blue, red, respectively). The solid line indicates the mean and dashed line indicates the median genomic heritability by instrument class.

750

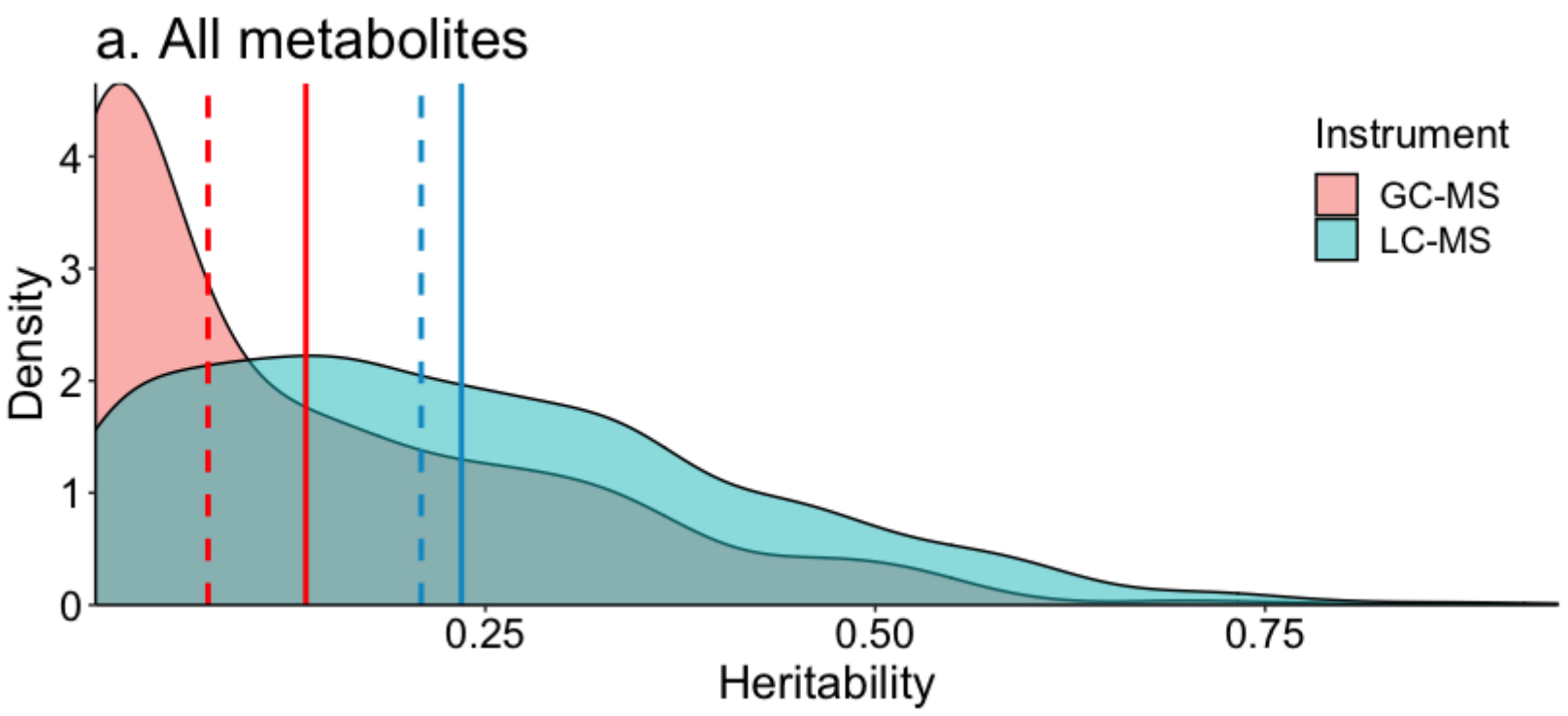

\section{b. Metabolites with GWAS result}

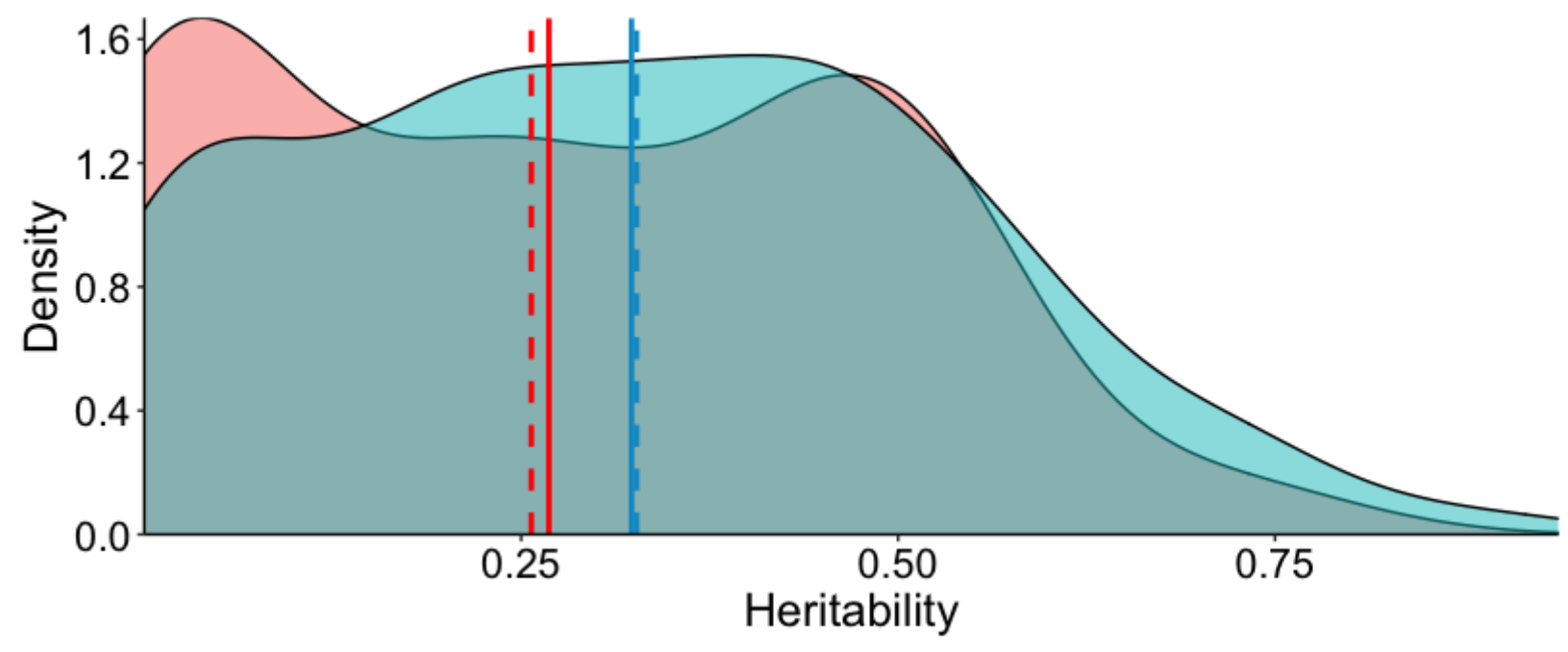


Figure 2. Distribution of metabolites by ClassyFire Superclass by general metabolite kernel in the discovery panel for (a) both LC-MS and GC-MS metabolites, (b) LC-MS metabolites only and (c) GC-MS metabolites only. Distributions of (d) LC-MS molecular mass, and (e) LC-MS and (f) GC-MS retention time ("RT") are shown by kernel. Significance indicators identify instances of depletion where $* p<0.05$, and $* * p<0.01$ and $* * * p<0.001$. Abbreviations of metabolite superclass are given in Table 1.
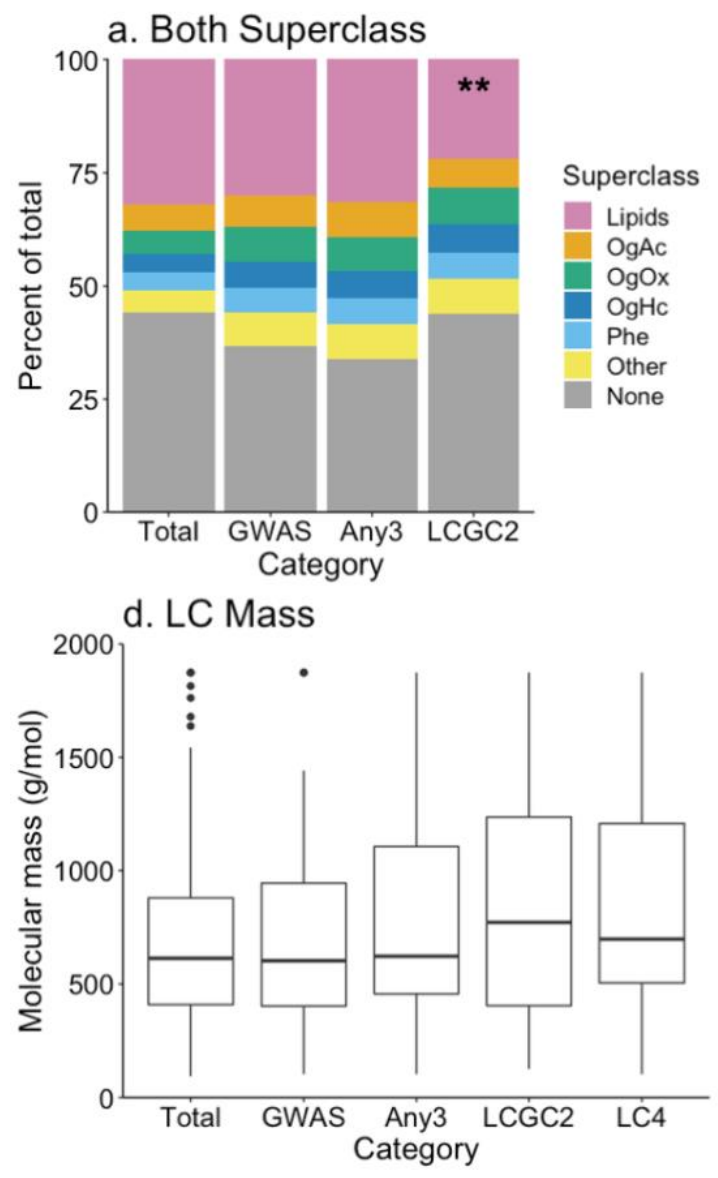

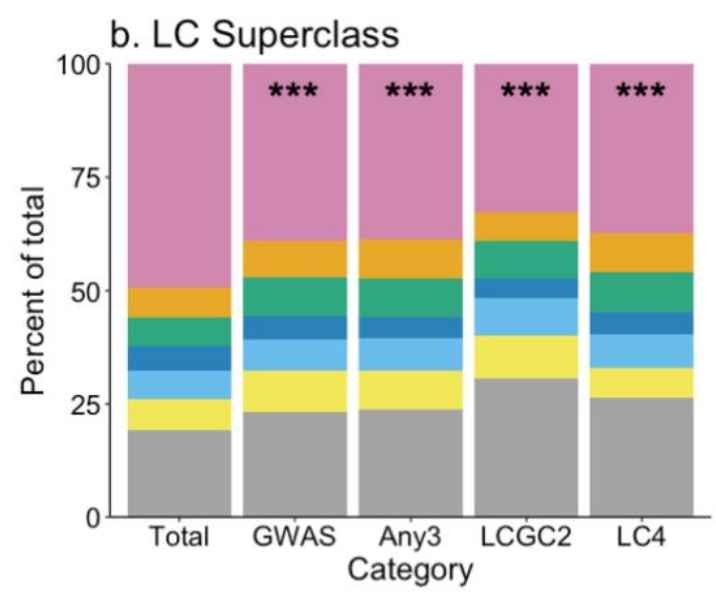

e. LC RT

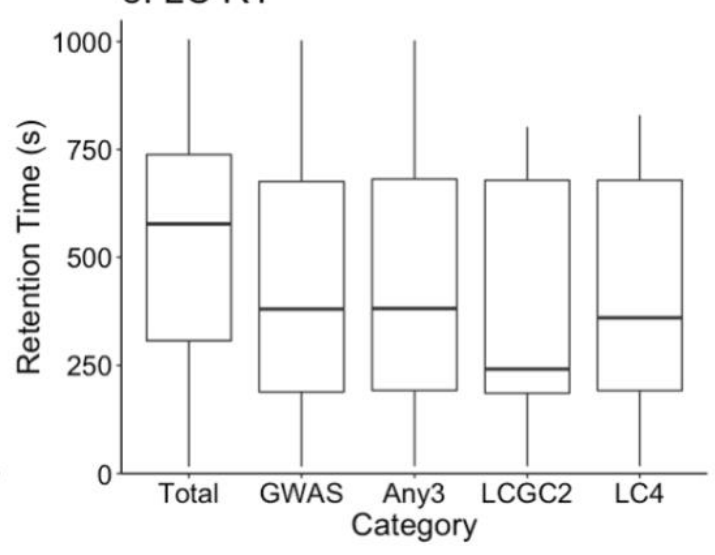

c. GC Superclass

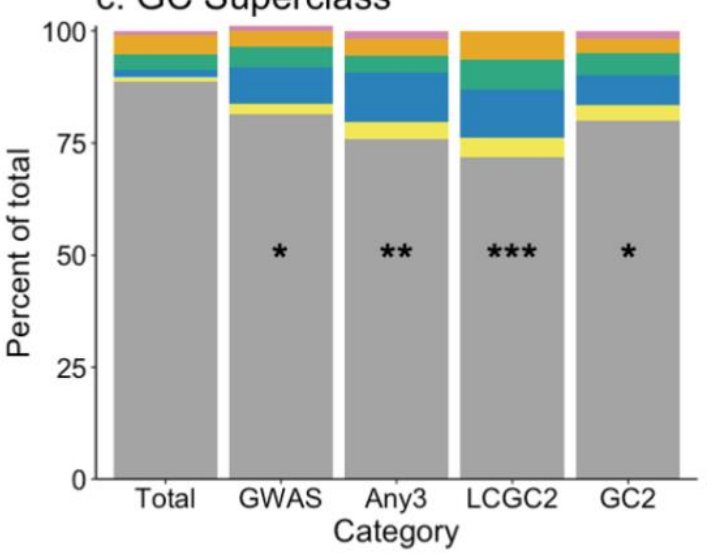

f. GC RT

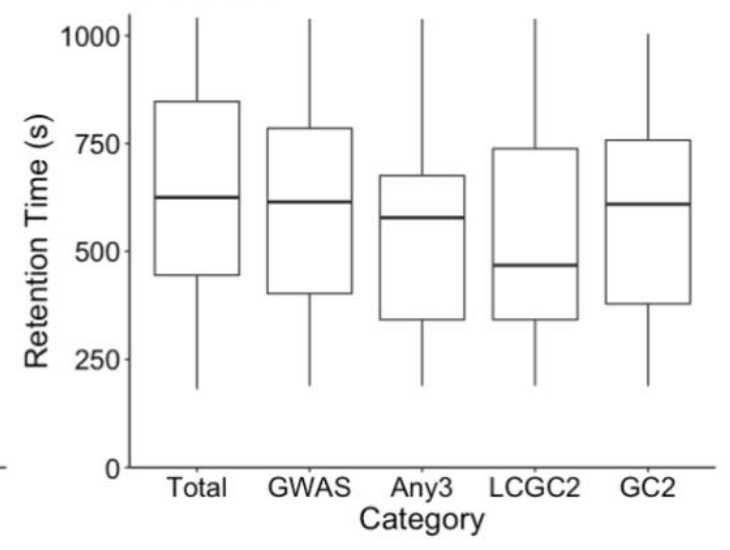


Figure 3. Mean cross-fold validation accuracy (r) of all (a.) LC-MS ( $n=397$ ) and (b.) GC-MS $(n=243)$ metabolites by environment (Minnesota, "MN"; South Dakota, "SD" and Wisconsin, "WI") and two-kernel metabolite model (see Table 2). The models were compared to GBLUP and significant difference indicators are given if the two-kernel metabolite model had higher accuracy than GBLUP at the top of the boxplot, and significance indicators of lower accuracy than GBLUP are given below. The * indicates a $p$-value less than the Bonferroni cutoff per plot, and $* *$ and $* * *$ indicate $\mathrm{p}<1 \mathrm{e}-4$, and $\mathrm{p}<1 \mathrm{e}-6$, respectively.

a. LC-MS

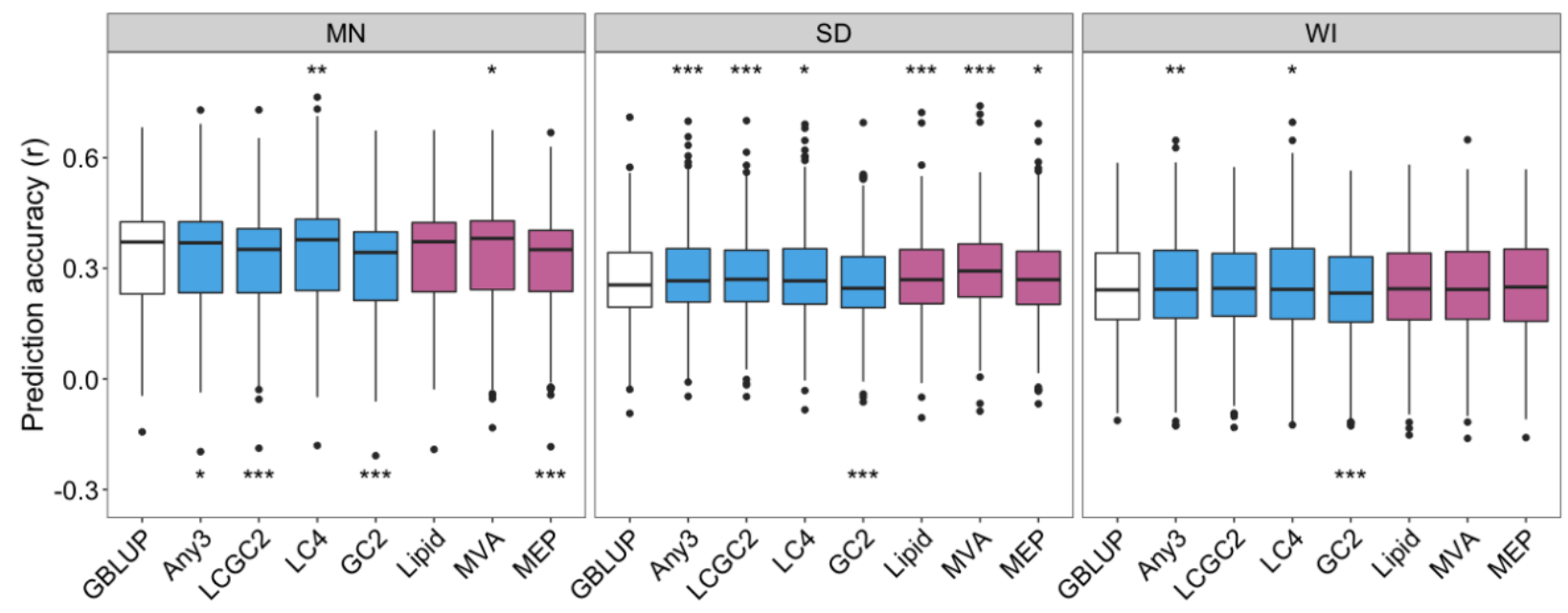

b. GC-MS

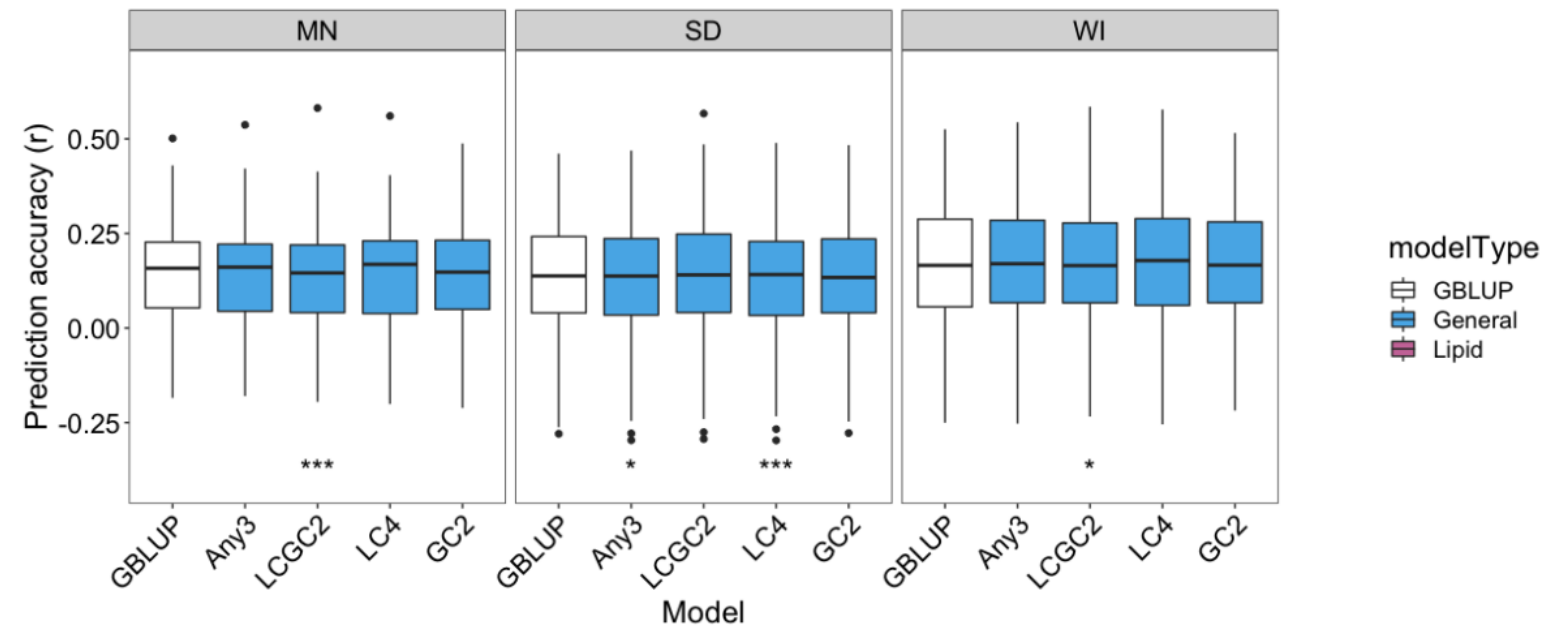


Figure 4. Percent genetic variation attributed to the metabolite kernel for LC-MS $(n=397)$ and GC-MS ( $n=243)$ metabolites in all environments (Minnesota, "MN"; South Dakota, "SD" and Wisconsin, "WI"). (a) The difference in percent genetic variation attributed to metabolite kernel between LC-MS and GC-MS metabolites, where significance indicators above the boxplot represent if percent variation is greater for LC-MS metabolites and below the boxplot if percent variation is greater for GC-MS metabolites. The difference between environments for (b) all metabolite models for LC-MS and (c) all general models for GC-MS instrument. The * indicates a $p$-value less than the Bonferroni cutoff per plot, and ** and *** indicate $\mathrm{p}<1 \mathrm{e}-4$, and $\mathrm{p}<1 \mathrm{e}-6$, respectively.

\section{a. By instrument}

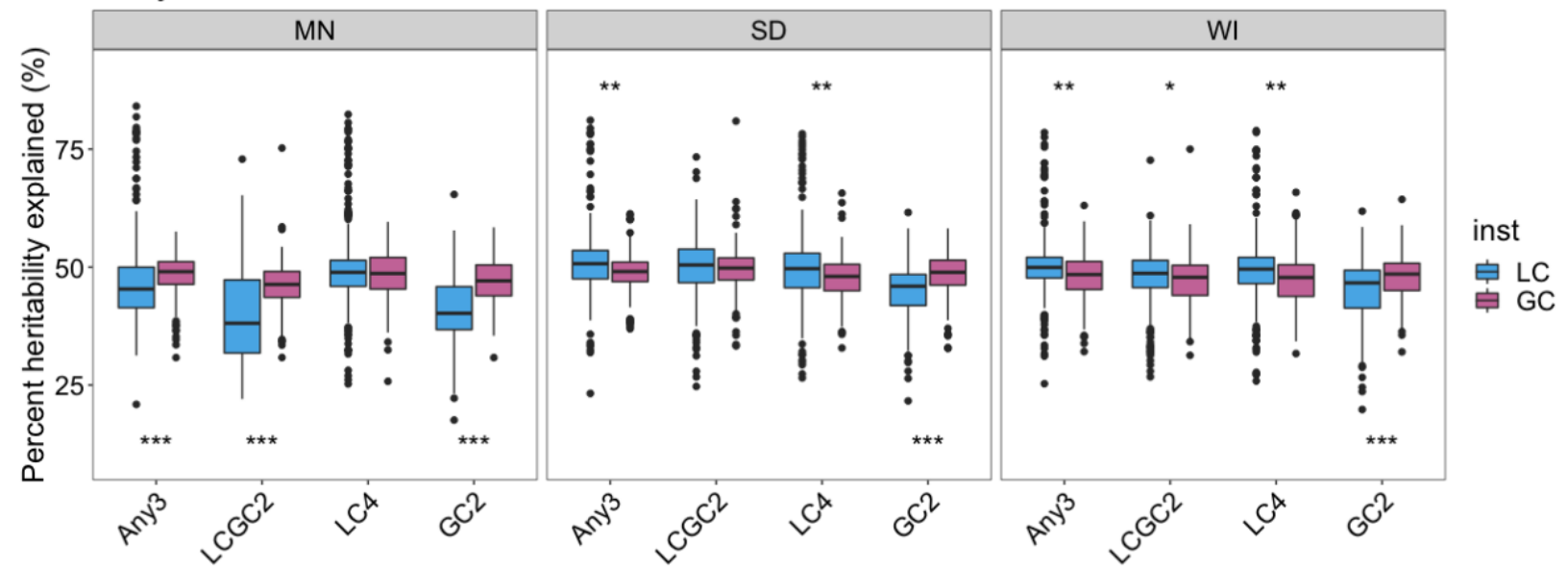

b. LCMS by environment

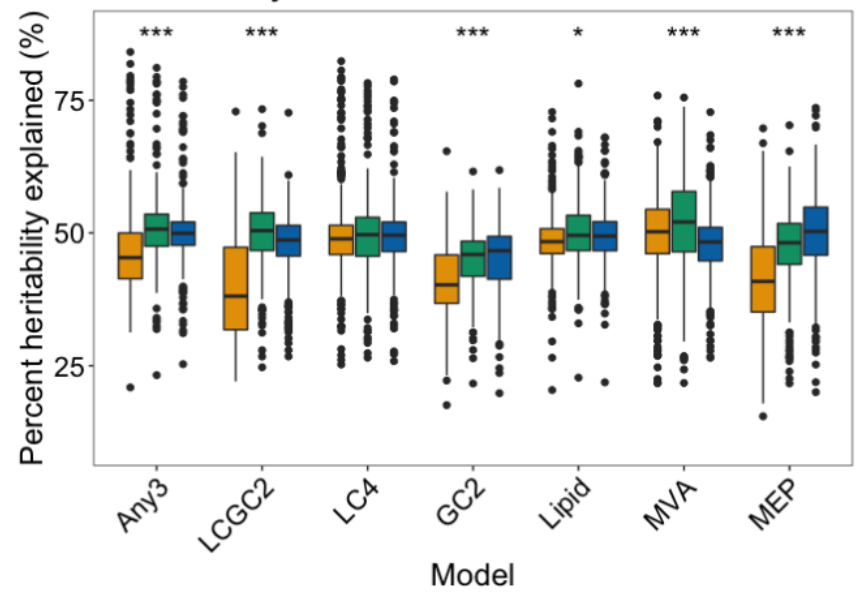

c. GCMS by environment

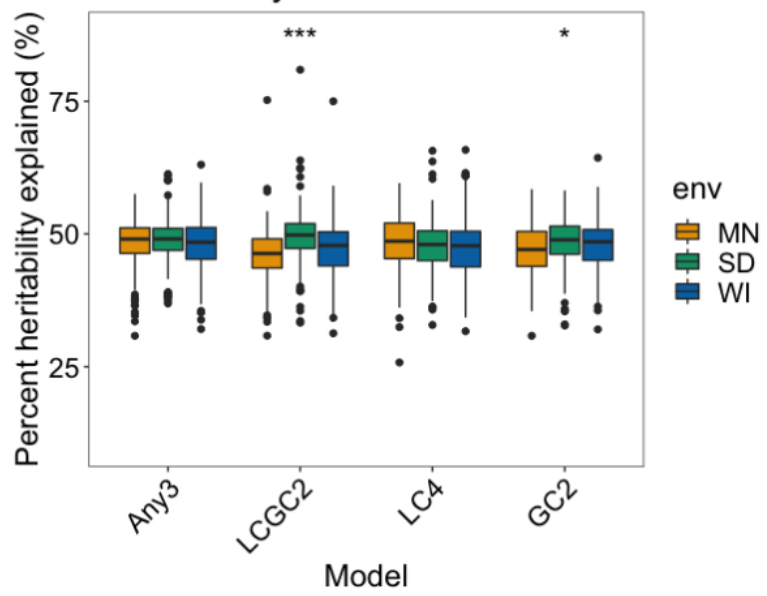


Figure 5. Dendrograms of distance in metabolite kernel performance for (a.-c.) LC-MS ( $n=397$ ) and (d.-f.) GC-MS ( $n=243$ ) metabolites by environment (Minnesota, "MN"; South Dakota, "SD" and Wisconsin, "WI”). Four hierarchical clusters are indicated by color and dashed box.

a. LC-MS, MN

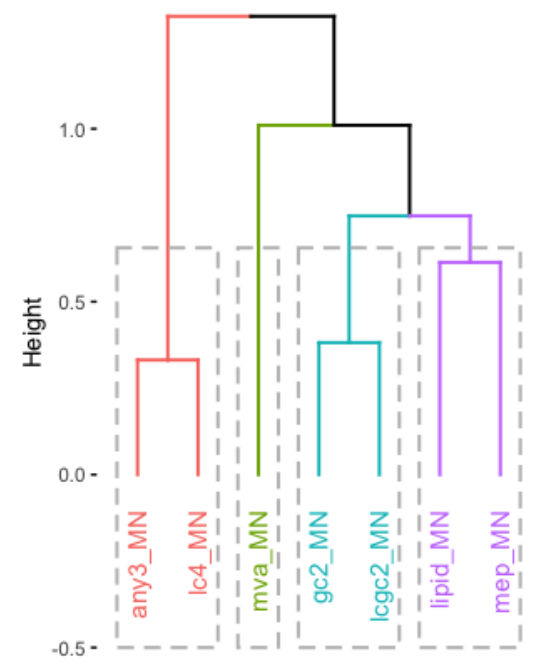

d. GC-MS, MN

0.75 -

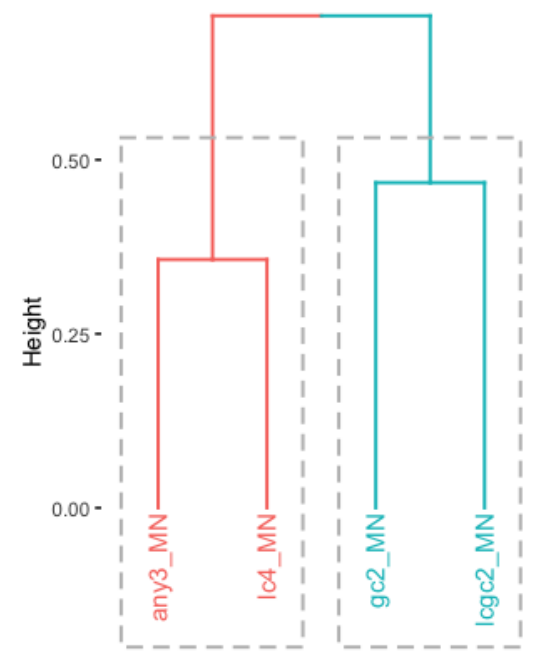

b. LC-MS, SD

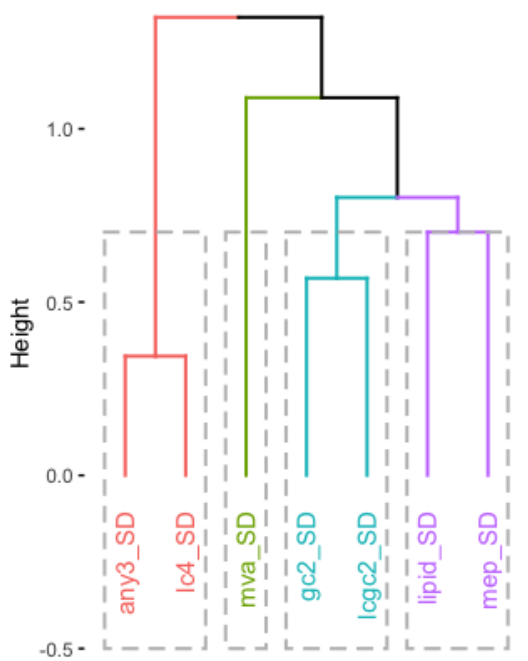

e. GC-MS, SD

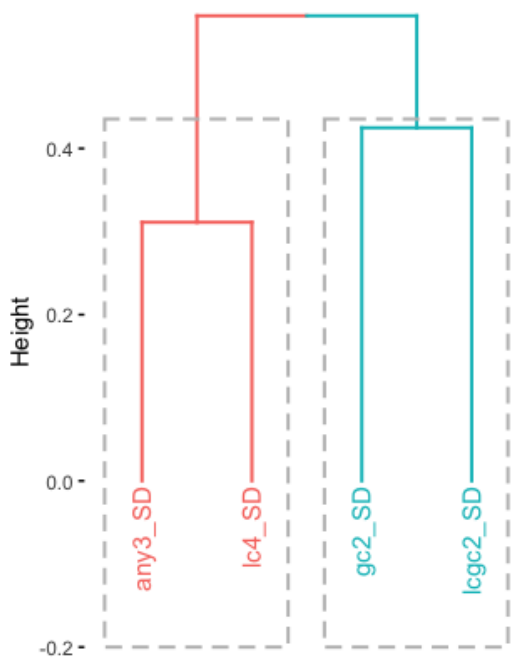

c. LC-MS, WI

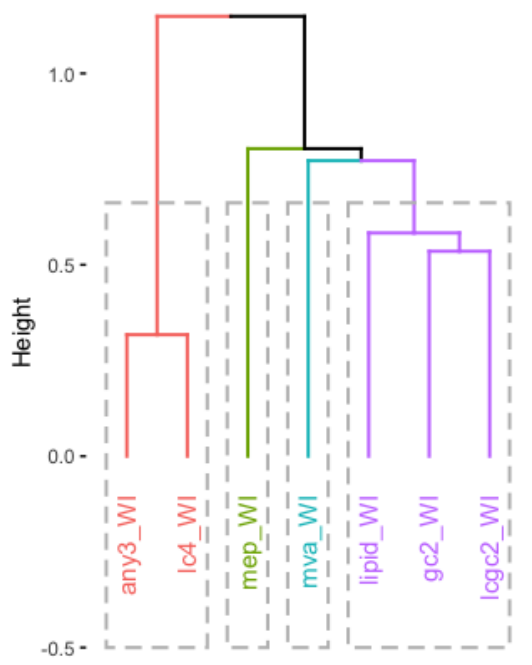

f.GC-MS, WI

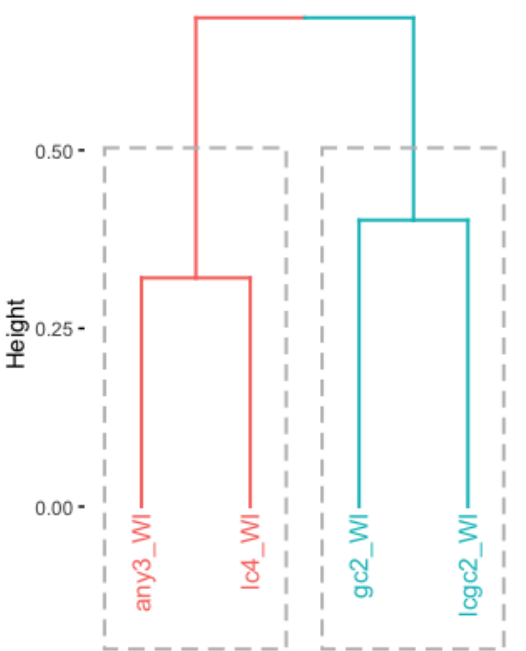


Figure 6. Correlograms of metabolite kernel prediction accuracy rank correlation for seven oat specialized metabolites by (a.) all environments together, and (b.-d.) by individual environment. A color indicator of correlation is shown for all correlations. The yellow boxes represent hierarchical clustering for $n=3$. The metabolite abbreviations are as follows: AVN_A, avenanthramide A; AVN_B, avenanthramide B; AEC_A1, AEC_A2, avenacin A1; AOS_A, avenacoside A; AOS_dA, 26-Desglucoavenacoside A; AOS_B, avenacoside B.

\section{a. All locations}

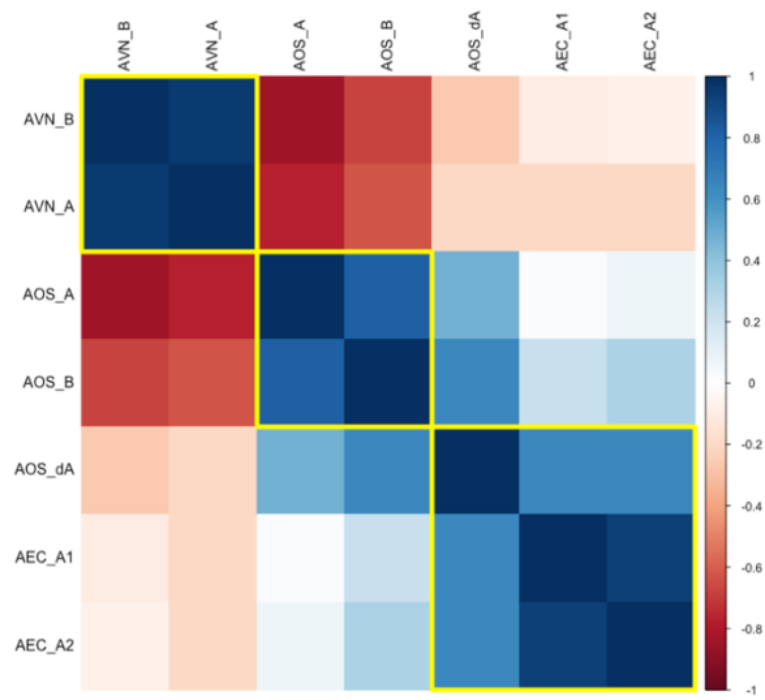

c. South Dakota

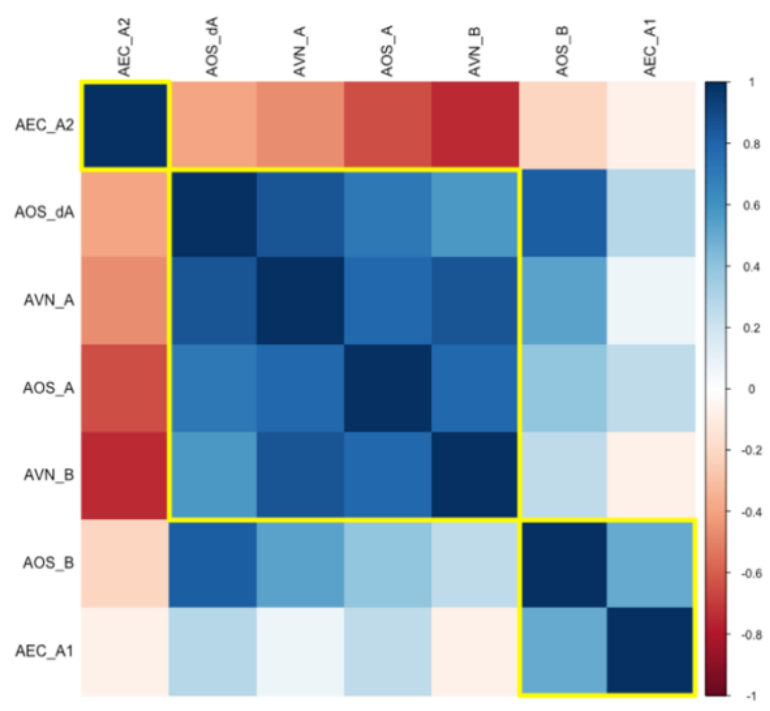

\section{b. Minnesota}

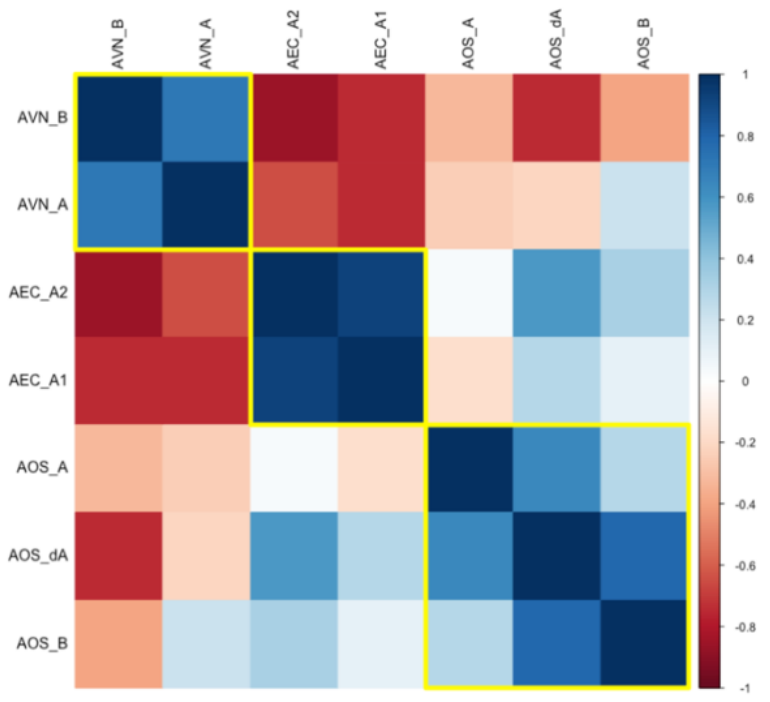

d. Wisconsin

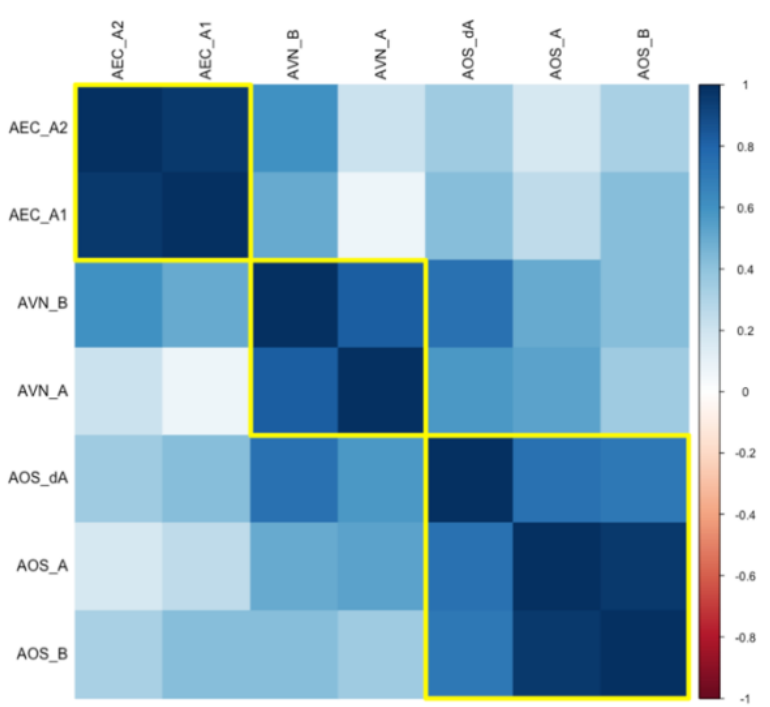


Figure 7. (a) Mean cross-fold validation accuracy (r) of, and (b) percent heritability (genetic variation) attributed to the metabolite kernel for, LC-MS lipid metabolites $(n=91)$ by environment (Minnesota, "MN"; South Dakota, "SD" and Wisconsin, "WI") and two-kernel metabolite model (see Table 2). The models were compared to 'Lipid' kernel and significant difference indicators are given if the two-kernel metabolite model had higher accuracy than 'Lipid' at the top of the boxplot, and significance indicators of lower accuracy than 'Lipid' are given below. The * indicates a $p$-value less than the Bonferroni cutoff per plot, and $* *$ and $* * *$ indicate $\mathrm{p}<1 \mathrm{e}-4$, and $\mathrm{p}<1 \mathrm{e}-6$, respectively.

a. Prediction accuracy comparison

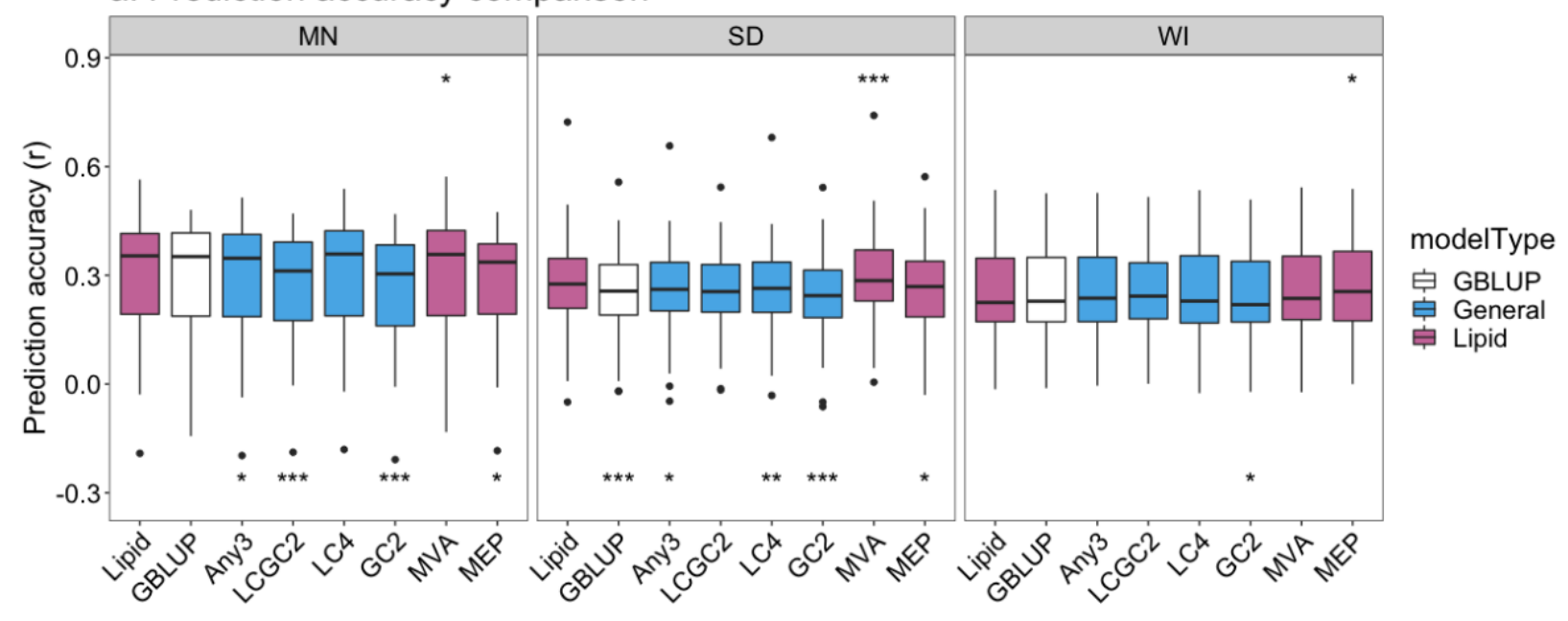

b. Percent heritability comparison

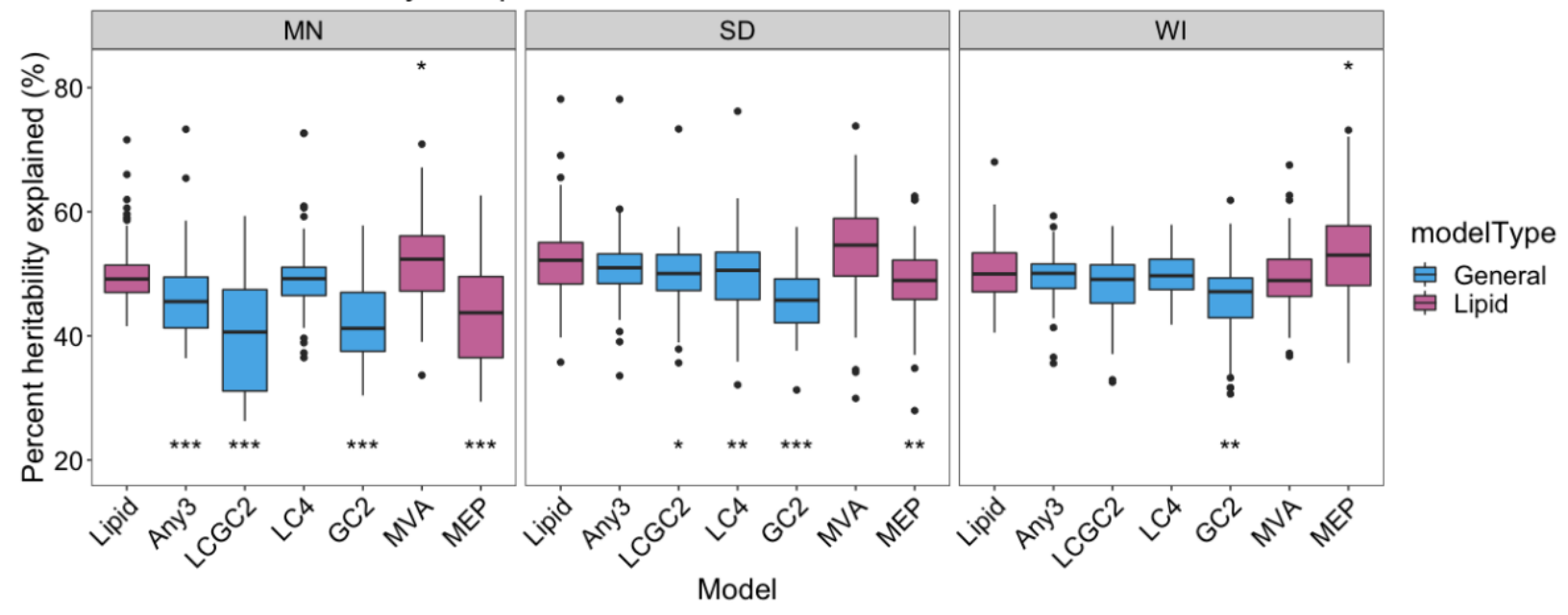


Figure 8. Correlograms of metabolite kernel prediction accuracy rank correlation by model for $n=91$ LC-MS lipids by (a.) all environments together, and by individual environment (b.-d.). A color indicator of correlation is shown for all correlations with $p<0.05$. The text label color indicates type of lipid. The yellow boxes represent hierarchical clustering for $n=6$. The name and color key for lipid type is given in Table $\mathbf{S 9 .}$

\section{a. Minnesota}

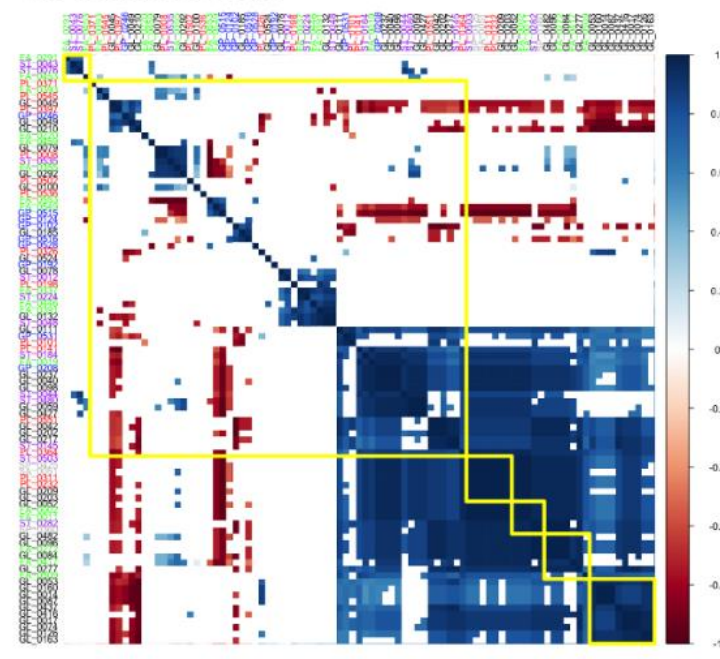

\section{c. Wisconsin}

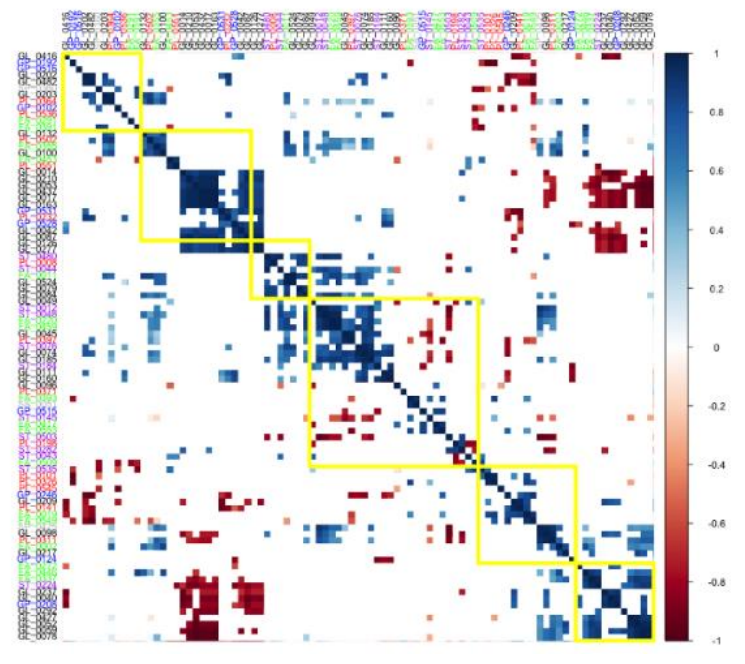

\section{b. South Dakota}

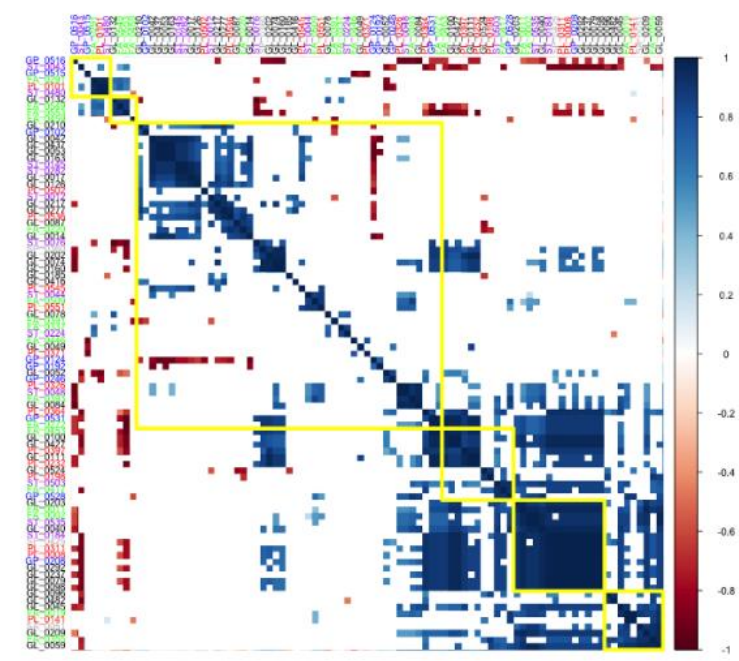


Figure 9. Rank correlation of metabolite kernel prediction accuracy rank correlation by model for groups of LC-MS metabolites defined by hierarchical clustering of a distance metric by environment (Minnesota, "MN"; South Dakota, "SD” and Wisconsin, "WI"). There is no relationship between cluster names across environments. Clusters with 10 or more metabolites are presented with the metabolites within the cluster are shown in blue, and the metabolites not in the cluster are shown in red, and comparisons are made between the two sets by group.

Significant difference indicators are given at the top of the boxplot if the metabolites within the group had stronger correlation than those not in the group, and vice versa for significance indicators below. The $*$ indicates a $p$-value less than the Bonferroni cutoff per plot, and $* *$ and $* * *$ indicate $\mathrm{p}<1 \mathrm{e}-4$, and $\mathrm{p}<1 \mathrm{e}-6$, respectively.

\section{a. MN, LC-MS}

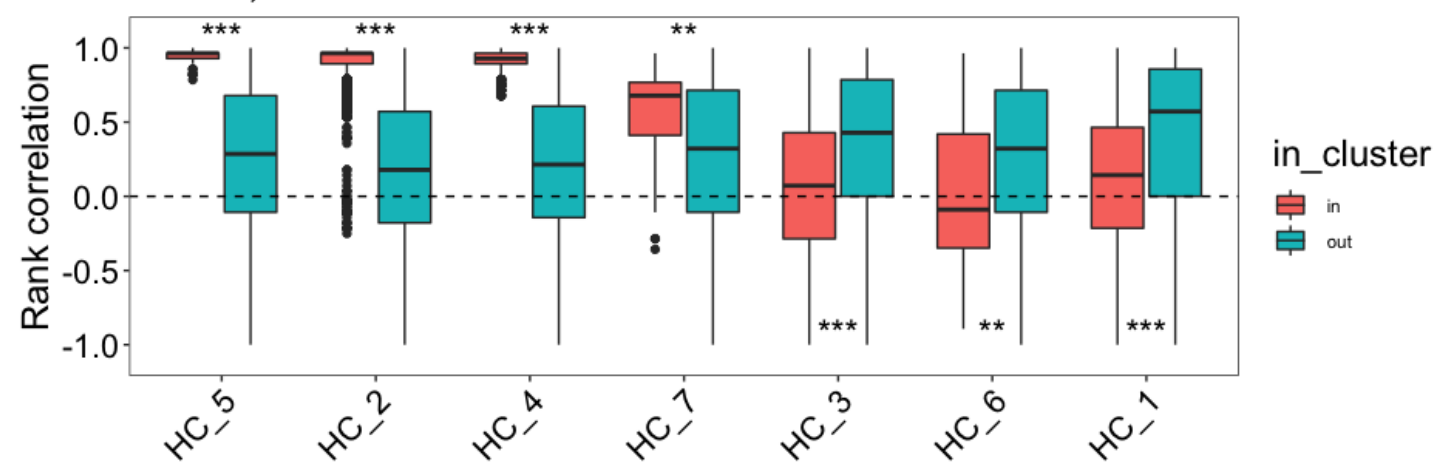

b. SD, LC-MS

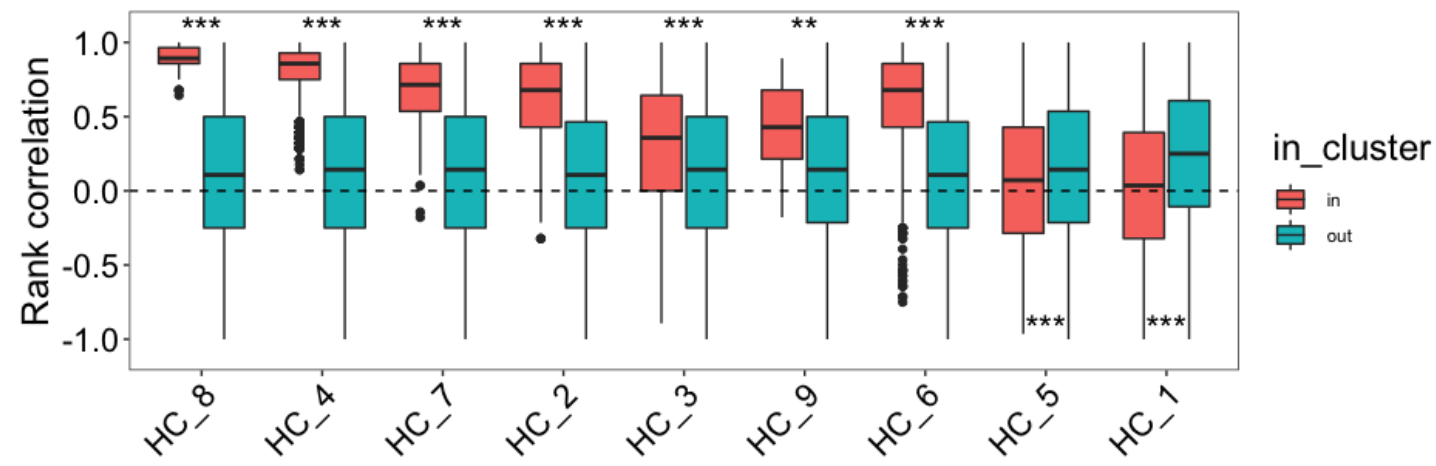

c. WI, LC-MS

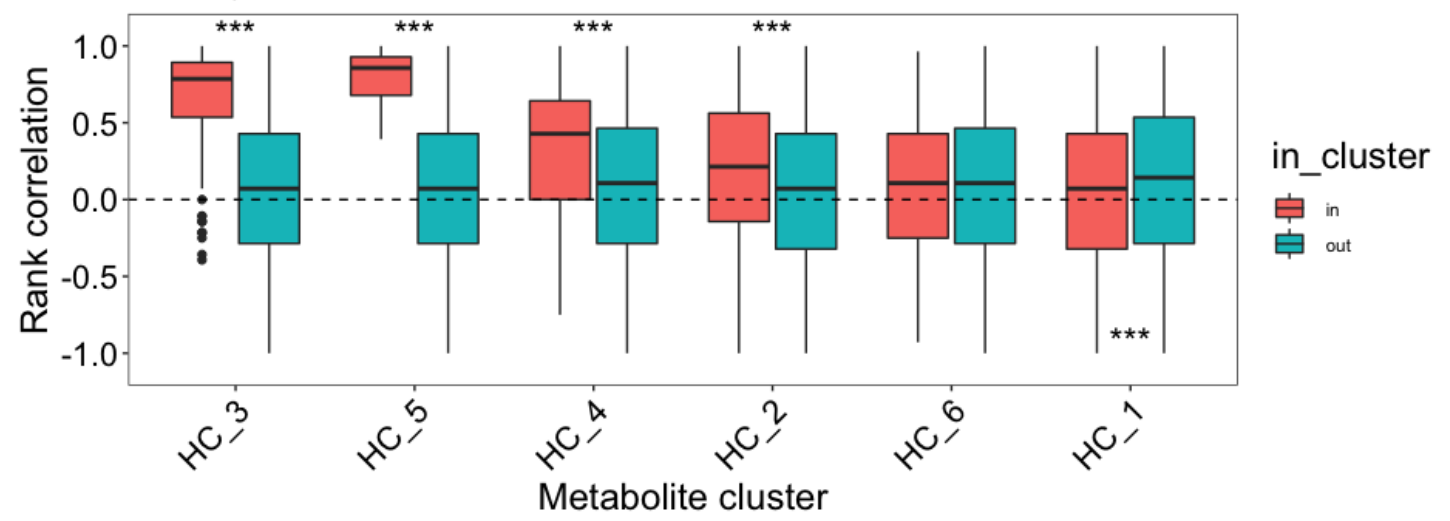


Table 1. Metabolite classification of the discovery panel for categorical variables of ClassyFire superclass and class, and numeric metrics of retention time and molecular mass. The distribution of metabolite retention time and molecular mass are given in Figure S1.

\begin{tabular}{|c|c|c|c|}
\hline Classification & LC & $\mathrm{GC}$ & Total \\
\hline \multicolumn{4}{|l|}{ ClassyFire Classification (count) } \\
\hline Lipids and lipid-like molecules ("Lipids") & 527 & 6 & 533 \\
\hline ...Glycerophospholipids & 123 & 1 & 124 \\
\hline ...Glycerolipids & 87 & 0 & 87 \\
\hline ...Fatty Acyls & 99 & 5 & 104 \\
\hline ...Steroids and steroid derivatives & 83 & 0 & 83 \\
\hline ...Prenol lipids & 102 & 0 & 102 \\
\hline Organoheterocyclic compounds ("OgHc" & 57 & 9 & 66 \\
\hline Phenylpropanoids and polyketides ("Phe") & 67 & 2 & 69 \\
\hline ...Cinnamic acids and derivatives & 13 & 1 & 14 \\
\hline ...Coumarins & 11 & 0 & 11 \\
\hline Organic acids and derivatives ("OgAc") & 70 & 26 & 96 \\
\hline ...Carboxylic acids and derivatives & 41 & 22 & 63 \\
\hline Organic oxygen compounds ("OgOx") & 68 & 20 & 88 \\
\hline Other ${ }^{1}$ & 74 & 6 & 80 \\
\hline Not classified ("None") & 204 & 532 & 736 \\
\hline \multicolumn{4}{|l|}{ Numeric metrics (mean) } \\
\hline Retention time (s) & 515.4 & 731.6 & 593.3 \\
\hline Molecular mass $(\mathrm{g} / \mathrm{mol})$ & 669.8 & NA & NA \\
\hline
\end{tabular}

${ }^{1}$ The 'Other' classification includes the nucleosides, nucleotides and analogues, and organic nitrogen compounds superclasses for all metabolites, and the alkaloids and derivatives, hydrocarbons, lignans, neolignans and related compounds, organic polymers and organosulfur compounds, and benzenoids superclasses for LC metabolites and homogenous non-metal for GC metabolites 
Table 2. Description and prediction of performance of metabolite kernels. The groups "MEP" and "MVA" refer to the Methylerythritol Phosphate pathway and Mevalonate Acid pathway branches of terpenoid biosynthesis, respectively.

\begin{tabular}{|c|c|c|c|c|}
\hline Type & Group & Description & Rationale & Predictions \\
\hline \multirow[t]{4}{*}{$\begin{array}{l}\text { General } \\
\text { metabolome }\end{array}$} & Any3 & $\begin{array}{l}\text { GWAS results shared by any } \\
\text { three or more metabolites (LC } \\
\text { or GC) }\end{array}$ & $\begin{array}{l}\text { Metabolites from either instrument, } \\
\text { extraction method contribute equally to } \\
\text { capturing broader metabolome variation }\end{array}$ & \multirow{2}{*}{$\begin{array}{l}\text { Since both instruments are } \\
\text { included, will perform best } \\
\text { for a broad range of } \\
\text { metabolites }\end{array}$} \\
\hline & LCGC2 & $\begin{array}{l}\text { GWAS results shared by at } \\
\text { least one LC and at least one } \\
\text { GC metabolite }\end{array}$ & $\begin{array}{l}\text { Including metabolites from both } \\
\text { instruments, extraction methods, is } \\
\text { necessary to capture metabolome variation }\end{array}$ & \\
\hline & LC4 & $\begin{array}{l}\text { GWAS results shared by four } \\
\text { or more LC metabolites }\end{array}$ & $\begin{array}{l}\text { Metabolites from a single instrument, } \\
\text { extraction method, but not restricted to a } \\
\text { specific class }\end{array}$ & \multirow{2}{*}{$\begin{array}{l}\text { Will perform better for } \\
\text { metabolites from respective } \\
\text { instruments, but will still } \\
\text { perform well for a broad } \\
\text { range of metabolites }\end{array}$} \\
\hline & GC2 & $\begin{array}{l}\text { GWAS results shared by two } \\
\text { or more GC metabolites }\end{array}$ & $\begin{array}{l}\text { Metabolites from a single instrument, } \\
\text { extraction method, but not restricted to a } \\
\text { specific class }\end{array}$ & \\
\hline \multirow[t]{2}{*}{ Lipids } & Lipid & $\begin{array}{l}\text { GWAS results shared by two } \\
\text { or more LC lipids }\end{array}$ & $\begin{array}{l}\text { Metabolites from a single instrument, } \\
\text { extraction, restricted to lipids }\end{array}$ & \multirow{2}{*}{$\begin{array}{l}\text { Will perform well for lipids, } \\
\text { but increased specificity } \\
\text { (MVA, MEP) will reduce } \\
\text { performance for metabolites } \\
\text { overall }\end{array}$} \\
\hline & $\begin{array}{l}\text { MVA, and } \\
\text { MEP }\end{array}$ & $\begin{array}{l}\text { GWAS results of any LC } \\
\text { MVA- or MEP-derived } \\
\text { terpenoids }\end{array}$ & $\begin{array}{l}\text { Metabolites from a single instrument, } \\
\text { extraction method, restricted to specific } \\
\text { biosynthetic pathways of terpenoids }\end{array}$ & \\
\hline
\end{tabular}


Table 3. Number of genes associated with each metabolite kernel. Kernel size is given in Table S3. The total genes implicated ('total genes'), the number of genes per SNP in the kernel ('genes per SNP'), and percent of SNPs in kernel in a gene ('Percent SNPs with a gene') are shown.

\begin{tabular}{llll}
\hline Kernel & Total genes & Genes per SNP & Percent SNPs within gene \\
\hline MVA & 127 & 0.127 & 11.01 \\
LC4 & 261 & 0.101 & 8.63 \\
Lipid & 225 & 0.092 & 8.07 \\
Any3 & 455 & 0.086 & 7.72 \\
MEP & 53 & 0.085 & 6.77 \\
GC2 & 150 & 0.072 & 6.80 \\
LCGC2 & 183 & 0.071 & 6.67 \\
\hline
\end{tabular}


Table 4. Mean cross-fold validation accuracy (r) of all LC-MS $(n=397)$ and GC-MS $(n=243)$ metabolites by (Minnesota, "MN"; South Dakota, "SD" and Wisconsin, "WI") and model (see Table 2). The color indicates relative value, where blue are highest values and red are lowest values, coded by instrument.

\begin{tabular}{|c|c|c|c|c|}
\hline Environment & \multicolumn{2}{|c|}{ Model } & LCMS & GCMS \\
\hline \multirow{8}{*}{ MN } & \multicolumn{2}{|c|}{ GBLUP } & 0.325 & 0.138 \\
\hline & \multirow{4}{*}{ General } & Any3 & 0.329 & 0.137 \\
\hline & & LCGC2 & 0.313 & 0.132 \\
\hline & & LC4 & 0.336 & 0.140 \\
\hline & & GC2 & 0.303 & 0.137 \\
\hline & \multirow{3}{*}{ Lipid } & Lipid & 0.326 & NA \\
\hline & & MEP & 0.314 & NA \\
\hline & & MVA & 0.334 & NA \\
\hline \multirow{8}{*}{ SD } & \multicolumn{2}{|c|}{ GBLUP } & 0.268 & 0.138 \\
\hline & \multirow{4}{*}{ General } & Any3 & 0.280 & 0.135 \\
\hline & & LCGC2 & 0.278 & 0.131 \\
\hline & & LC4 & 0.278 & 0.141 \\
\hline & & GC2 & 0.261 & 0.136 \\
\hline & \multirow{3}{*}{ Lipid } & Lipid & 0.278 & NA \\
\hline & & MEP & 0.273 & NA \\
\hline & & MVA & 0.296 & NA \\
\hline \multirow{8}{*}{ WI } & \multicolumn{2}{|c|}{ GBLUP } & 0.249 & 0.167 \\
\hline & \multirow{4}{*}{ General } & Any3 & 0.256 & 0.167 \\
\hline & & LCGC2 & 0.251 & 0.163 \\
\hline & & LC4 & 0.256 & 0.172 \\
\hline & & GC2 & 0.239 & 0.168 \\
\hline & \multirow{3}{*}{ Lipid } & Lipid & 0.248 & NA \\
\hline & & MEP & 0.250 & NA \\
\hline & & MVA & 0.250 & NA \\
\hline
\end{tabular}


Table 5. Number of metabolites (of 397 LC-MS and 243 GC-MS metabolites) where the crossfold validation accuracy (r) of the given metabolite model (see Table 2) is significantly greater or less than the accuracy of GBLUP. The environments are: Minnesota, "MN", South Dakota, "SD" and Wisconsin, "WI". The color indicates relative value, where blue are highest values and red are lowest values, coded by column.

\begin{tabular}{|c|c|c|c|c|c|c|}
\hline \multirow{2}{*}{ Type } & \multirow{2}{*}{ Model } & \multirow{2}{*}{ Env } & \multicolumn{2}{|c|}{ LCMS } & \multicolumn{2}{|c|}{ GCMS } \\
\hline & & & n_better & n_worse & n_better & n_worse \\
\hline \multirow{12}{*}{ General } & \multirow{3}{*}{ Any3 } & $\mathrm{MN}$ & 39 & 24 & 29 & 40 \\
\hline & & SD & 59 & 14 & 15 & 31 \\
\hline & & WI & 31 & 20 & 31 & 23 \\
\hline & \multirow{3}{*}{ LCGC2 } & $\mathrm{MN}$ & 7 & 158 & 31 & 33 \\
\hline & & SD & 61 & 16 & 12 & 18 \\
\hline & & WI & 36 & 22 & 26 & 18 \\
\hline & \multirow{3}{*}{ LC4 } & $\mathrm{MN}$ & 41 & 27 & 36 & 44 \\
\hline & & SD & 55 & 41 & 13 & 40 \\
\hline & & $\mathrm{WI}$ & 30 & 30 & 39 & 26 \\
\hline & \multirow{3}{*}{ GC2 } & $\mathrm{MN}$ & 6 & 122 & 16 & 39 \\
\hline & & SD & 9 & 41 & 19 & 17 \\
\hline & & WI & 8 & 62 & 16 & 35 \\
\hline \multirow{9}{*}{ Lipid } & \multirow{3}{*}{ Lipid } & $\mathrm{MN}$ & 20 & 22 & NA & NA \\
\hline & & SD & 64 & 20 & NA & NA \\
\hline & & WI & 18 & 27 & NA & NA \\
\hline & \multirow{3}{*}{ MEP } & $\mathrm{MN}$ & 20 & 104 & NA & NA \\
\hline & & SD & 53 & 30 & NA & NA \\
\hline & & $\mathrm{WI}$ & 40 & 43 & NA & NA \\
\hline & \multirow{3}{*}{ MVA } & $\mathrm{MN}$ & 44 & 29 & $\mathrm{NA}$ & NA \\
\hline & & SD & 133 & 20 & NA & NA \\
\hline & & WI & 32 & 36 & NA & NA \\
\hline
\end{tabular}


Table 6. Coefficient of variation ("CV") in retention time (s) and genomic heritability (mean +/- one standard deviation) of LC-MS metabolites by metabolite group defined by hierarchical cluster. Note that there is no relationship between cluster name across environments. The number of metabolites in each group is given by ' $n$ '. Metabolite groups with ten or more metabolites that had higher within group correlation are indicated with a *.

\begin{tabular}{|c|c|c|c|c|c|c|c|c|c|c|c|c|}
\hline \multirow{2}{*}{ Group } & \multicolumn{4}{|c|}{$\mathrm{MN}$} & \multicolumn{4}{|c|}{ SD } & \multicolumn{4}{|c|}{ WI } \\
\hline & $n$ & $R T-C V$ & $h^{2}$ & & $n$ & $R T-C V$ & $h^{2}$ & & $n$ & $R T-C V$ & $h^{2}$ & \\
\hline 1 & 134 & 80.2 & $0.30+/-0.19$ & & 123 & 87.7 & $0.22+/-0.19$ & & 206 & 70.5 & $0.20+/-0.15$ & \\
\hline 2 & 90 & 11.2 & $0.39+/-0.07$ & $*$ & 29 & 2.8 & $0.09+/-0.06$ & $*$ & 84 & 13.4 & $0.11+/-0.06$ & $*$ \\
\hline 3 & 69 & 24.2 & $0.17+/-0.12$ & & 44 & 21.8 & $0.24+/-0.17$ & $*$ & 28 & 2.7 & $0.11+/-0.05$ & $*$ \\
\hline 4 & 54 & 3.2 & $0.42+/-0.04$ & $*$ & 47 & 2.7 & $0.10+/-0.03$ & $*$ & 26 & 3.2 & $0.08+/-0.04$ & $*$ \\
\hline 5 & 16 & 1.4 & $0.40+/-0.02$ & $*$ & 52 & 25.7 & $0.21+/-0.15$ & & 11 & 6.6 & $0.39+/-0.07$ & $*$ \\
\hline 6 & 12 & 10.1 & $0.08+/-0.07$ & & 54 & 15.8 & $0.12+/-0.06$ & $*$ & 31 & 24.7 & $0.18+/-0.14$ & \\
\hline 7 & 11 & 6.3 & $0.10+/-0.06$ & $*$ & 21 & 2.0 & $0.07+/-0.04$ & $*$ & 4 & 1.3 & $0.22+/-0.03$ & NA \\
\hline 8 & 5 & 9.6 & $0.18+/-0.16$ & NA & 11 & 6.6 & $0.26+/-0.04$ & $*$ & 3 & 7.4 & $0.20+/-0.06$ & NA \\
\hline 9 & 3 & 7.4 & $0.14+/-0.08$ & NA & 10 & 6.5 & $0.10+/-0.04$ & $*$ & 2 & 2.8 & $0.48+/-0.13$ & NA \\
\hline 10 & 2 & 2.8 & $0.47+/-0.07$ & NA & 5 & 1.3 & $0.19+/-0.06$ & NA & 1 & NA & NA & NA \\
\hline
\end{tabular}

